\title{
Wave propagation in anisotropic elastic materials and curvilinear coordinates using a summation-by-parts finite difference method
}

\author{
N. Anders Petersson ${ }^{1}$, Björn Sjögreen \\ Center for Applied Scientific Computing, Lawrence Livermore National Laboratory, PO \\ Box 808, Livermore CA 94551.
}

\begin{abstract}
We develop a fourth order accurate finite difference method for solving the three-dimensional elastic wave equation in general heterogeneous anisotropic materials on curvilinear grids. The proposed method is an extension of the method for isotropic materials, previously described in the paper by Sjögreen and Petersson [J. Sci. Comput. 52 (2012)]. The proposed method discretizes the anisotropic elastic wave equation in second order formulation, using a node centered finite difference method that satisfies the principle of summation by parts. The summation by parts technique results in a provably stable numerical method that is energy conserving. We also generalize and evaluate the super-grid far-field technique for truncating unbounded domains. Unlike the commonly used perfectly matched layers (PML), the super-grid technique is stable for general anisotropic material, because it is based on a coordinate stretching combined with an artificial dissipation. As a result, the discretization satisfies an energy estimate, proving that the numerical approximation is stable. We demonstrate by numerical experiments that sufficiently wide super-grid layers result in very small artificial reflections. Applications of the proposed method are demonstrated by three-dimensional simulations of anisotropic wave propagation in crystals.
\end{abstract}

Keywords: Anisotropy, elastic wave equation, curvilinear coordinates, far-field closure, summation-by-parts

Email addresses: petersson1@llnl.gov (N. Anders Petersson), sjogreen2@llnl.gov (Björn Sjögreen)

${ }^{1}$ Corresponding author 


\section{Introduction}

This paper describes a fourth order accurate numerical method for calculating wave propagation in general anisotropic elastic materials, i.e., materials in which waves propagate with different speeds in different directions. Such materials occur in several applications. One class of anisotropic materials are crystals. Here the directionally dependent wave propagation properties follow from the symmetries and structure of the atomic bonds in the crystal. In seismic applications, isotropic layered materials behave anisotropically when they are subjected to waves where the wavelength is much longer than the thickness of the layers [1]. Furthermore, fractures in an isotropic material can lead to directionally dependent wave propagation properties [2], i.e., anisotropic behavior. More generally, spatial homogenization of a fine grained heterogeneous isotropic elastic material is known to result in a coarser grained elastic model with anisotropic properties [3, 4].

Many wave propagation codes for isotropic elastic materials are based on finite difference methods on staggered grids $[5,6,7]$. These methods approximate the elastic wave equation in first order velocity-stress formulation. Unfortunately, the staggered grid approach is non-trivial to generalize to general anisotropic materials. The fundamental difficulty is to place the dependent variables on the staggered grid, such that all terms in the anisotropic Hooke's law can be approximated accurately and, at the same time, making the numerical method stable. Since an isotropic material has anisotropic properties when the equations are transformed to curvilinear coordinates, similar difficulties occur for staggered grid methods on curvilinear meshes. Node centered methods, which discretize the elastic wave equation in second order displacement formulation, do not have this difficulty. For example, the spectral element method, described in [8], is naturally formulated for general linear stress-strain relationships, and has successfully been used for modeling general anisotropy [9] as well as realistic topography using curvilinear (unstructured hexahedral) meshes [10].

The present paper has two objectives. First, we describe a fourth order accurate node centered finite difference scheme for wave propagation in general anisotropic elastic materials. Our scheme satisfies the principle of summation by parts (SBP) and is a generalization of the method described in $[11,12]$, which is implemented in the elastic wave propagation open source code SW4, version 1.0 [13]. The finite difference scheme is fourth order accurate, stable, and energy conserving. We here generalize the method to a fully anisotropic material in curvilinear coordinates, allowing for accurate modeling of realistic topography. Our main motivation for using the sum- 
mation by parts method is to obtain a spatial discretization that satisfies an energy estimate, which guarantees stability of the numerical approximation for heterogeneous materials on curvilinear grids with free surface or Dirichlet boundary conditions. We remark that our SBP method uses ghost points just outside the boundaries to enforce the boundary conditions in a strong (point-wise) sense. There is a related SBP method that does not use ghost points and instead enforces the boundary conditions in a weak sense using penalty techniques, see e.g. $[14,15,16]$.

The second objective is to analyze and numerically evaluate the supergrid far-field truncation technique for anisotropic elastic materials. Supergrid far-field conditions truncate very large or unbounded domains to finite extent by adding sponge layers outside the domain of interest. Inside the layers, the wave equation is modified by a combination of grid stretching and high order artificial dissipation. Compared to perfectly matched layers (PML) [17], the greatest strength of the super-grid technique is that the overall numerical method is provably stable, as long as the underlying numerical method is stable on a curvilinear grid. Note that the PML equations can have unstable solutions (growing exponentially in time) for some anisotropic materials [18] that violate the so-called geometric stability condition [19]. We have previously proven that the isotropic discretized elastic wave equation with super-grid layers satisfies an energy estimate [12], precluding exponential growth of the numerical solution. In the present paper, we extend that analysis to general anisotropic elastic materials on curvilinear grids. An additional strength of the super-grid technique is its simplicity and low computational cost. In contrast to the PML method, super-grid does not rely on augmenting the wave equation with additional differential equations that govern additional dependent variables. A potential weakness of the super-grid technique is that it does not achieve the 'perfect' nonreflecting property of PML. However, numerical experiments indicate that artificial reflections decay rapidly with the width of the super-grid layers.

This paper is organized as follows. In Section 2, we review the equations of anisotropic elastic wave propagation in Cartesian coordinates. Section 3 generalizes the results of Section 2 to curvilinear coordinates. The finite difference discretization is presented in Section 4, where we also present an efficient way of estimating the stability limit for the time step. Section 5 describes the super-grid technique and numerical experiments are presented in Section 6. Here we verify the accuracy of the proposed finite difference scheme, demonstrate the accuracy of the super-grid far-field truncation technique, and verify energy conservation of the numerical solution. Conclusions are given in Section 7. 


\section{The anisotropic elastic wave equation}

To make the presentation self-contained and to define a consistent notation, we start by introducing the governing equations in a form that is amenable for constructing the SBP discretization. For further background information, we recommend an advanced text book on solid mechanics, e.g. $[20]$.

We consider the time-dependent elastic wave equation in a three-dimensional domain $\mathbf{x} \in \Omega$, where $\mathbf{x}=\left(x^{(1)}, x^{(2)}, x^{(3)}\right)^{T}$ are the Cartesian coordinates and $\mathbf{u}=\left(u^{(1)}, u^{(2)}, u^{(3)}\right)^{T}$ are the Cartesian components of the three-dimensional displacement vector. In displacement formulation, the elastic wave equation takes the form

$$
\begin{aligned}
\rho \frac{\partial^{2} \mathbf{u}}{\partial t^{2}} & =\nabla \cdot \mathcal{T}+\mathbf{F}, \quad \mathbf{x} \in \Omega, \quad t \geq 0 \\
\nabla \cdot \mathcal{T} & =G_{s}^{T} C G_{s} \mathbf{u}=: \mathbf{L u},
\end{aligned}
$$

subject to appropriate initial and boundary conditions. Here, $\rho$ is the density, $\mathcal{T}$ is the stress tensor, and $\mathbf{F}$ is the external forcing per unit volume. The spatial operator $\mathbf{L}$ is called the $3 \times 3$ symmetric Kelvin-Christoffel differential operator matrix [20]. Let $\mathcal{T}_{i j}$ and $\mathcal{E}_{i j}$ be the Cartesian components of the symmetric stress and strain tensors, respectively. We adopt Voigt's vector notation,

$$
\boldsymbol{\sigma}=\left(\mathcal{T}_{11}, \mathcal{T}_{22}, \mathcal{T}_{33}, \mathcal{T}_{23}, \mathcal{T}_{13}, \mathcal{T}_{12}\right)^{T}, \quad \mathbf{e}=\left(\mathcal{E}_{11}, \mathcal{E}_{22}, \mathcal{E}_{33}, 2 \mathcal{E}_{23}, 2 \mathcal{E}_{13}, 2 \mathcal{E}_{12}\right)^{T}
$$

which allows Hooke's law to be expressed in terms of the $6 \times 6$ stiffness matrix $C$, which is symmetric and positive definite [20]. Because $C$ is symmetric, it has 21 unique elements, corresponding to the 21 parameters of a general anisotropic material. With this notation, the strain vector and the divergence of the stress tensor can be expressed in terms of the symmetric part of the gradient operator and its transpose, respectively,

$$
\mathbf{L u}=G_{s}^{T} \boldsymbol{\sigma}, \boldsymbol{\sigma}=C \mathbf{e}, \mathbf{e}=G_{s} \mathbf{u}, G_{s}^{T}=\left(\begin{array}{cccccc}
\partial_{1} & 0 & 0 & 0 & \partial_{3} & \partial_{2} \\
0 & \partial_{2} & 0 & \partial_{3} & 0 & \partial_{1} \\
0 & 0 & \partial_{3} & \partial_{2} & \partial_{1} & 0
\end{array}\right)
$$

where $\partial_{k}=\partial / \partial x^{(k)}$.

For the purpose of constructing a SBP discretization, we introduce the notation

$$
G_{s}^{T} \boldsymbol{\sigma}=P_{1}^{T} \partial_{1} \boldsymbol{\sigma}+P_{2}^{T} \partial_{2} \boldsymbol{\sigma}+P_{3}^{T} \partial_{3} \boldsymbol{\sigma}
$$


where the matrices are defined by

$$
P_{1}^{T}=\left(\begin{array}{llllll}
1 & 0 & 0 & 0 & 0 & 0 \\
0 & 0 & 0 & 0 & 0 & 1 \\
0 & 0 & 0 & 0 & 1 & 0
\end{array}\right), \quad P_{2}^{T}=\left(\begin{array}{llllll}
0 & 0 & 0 & 0 & 0 & 1 \\
0 & 1 & 0 & 0 & 0 & 0 \\
0 & 0 & 0 & 1 & 0 & 0
\end{array}\right),
$$

and

$$
P_{3}^{T}=\left(\begin{array}{llllll}
0 & 0 & 0 & 0 & 1 & 0 \\
0 & 0 & 0 & 1 & 0 & 0 \\
0 & 0 & 1 & 0 & 0 & 0
\end{array}\right)
$$

By using (4) in (2), we obtain

$$
\begin{aligned}
\mathbf{L u}=\sum_{j=1}^{3} P_{j}^{T} \partial_{j}\left(C \sum_{j=k}^{3} P_{k} \partial_{k} \mathbf{u}\right)=\sum_{j=1}^{3} \sum_{k=1}^{3} \partial_{j}\left(P_{j}^{T} C P_{k} \partial_{k} \mathbf{u}\right) \\
=: \sum_{j=1}^{3} \sum_{k=1}^{3} \partial_{j}\left(M^{j k} \partial_{k} \mathbf{u}\right)
\end{aligned}
$$

where the $3 \times 3$ matrices on the right hand side are defined by

$$
M^{j k}=P_{j}^{T} C P_{k} .
$$

Hence, each matrix $M^{j k}$ contains a subset of the elements of $C$, as determined by the matrices $P_{j}$. We refer to Appendix A for the exact expressions of $M^{j k}$. From the positive definiteness of $C$ it follows that $M^{11}, M^{22}$, and $M^{33}$ are also positive definite. Moreover, the definition (6) shows that $M^{k j}=\left(M^{j k}\right)^{T}$. With this notation we can write

$$
\mathbf{L u}=\partial_{1}\left(A_{1} \nabla \mathbf{u}\right)+\partial_{2}\left(A_{2} \nabla \mathbf{u}\right)+\partial_{3}\left(A_{3} \nabla \mathbf{u}\right)
$$

where

$$
\begin{aligned}
& A_{1} \nabla \mathbf{u}:=M^{11} \partial_{1} \mathbf{u}+M^{12} \partial_{2} \mathbf{u}+M^{13} \partial_{3} \mathbf{u}, \\
& A_{2} \nabla \mathbf{u}:=M^{21} \partial_{1} \mathbf{u}+M^{22} \partial_{2} \mathbf{u}+M^{23} \partial_{3} \mathbf{u}, \\
& A_{3} \nabla \mathbf{u}:=M^{31} \partial_{1} \mathbf{u}+M^{32} \partial_{2} \mathbf{u}+M^{33} \partial_{3} \mathbf{u}
\end{aligned}
$$

Because $\mathbf{L u}$ is equal to the divergence of the stress tensor, we also have

$$
A_{1} \nabla \mathbf{u}=\left(\begin{array}{c}
\mathcal{T}_{11} \\
\mathcal{T}_{12} \\
\mathcal{T}_{13}
\end{array}\right), \quad A_{2} \nabla \mathbf{u}=\left(\begin{array}{c}
\mathcal{T}_{12} \\
\mathcal{T}_{22} \\
\mathcal{T}_{23}
\end{array}\right), \quad A_{3} \nabla \mathbf{u}=\left(\begin{array}{c}
\mathcal{T}_{13} \\
\mathcal{T}_{23} \\
\mathcal{T}_{33}
\end{array}\right)
$$


From (11) it follows that a boundary with unit normal $\mathbf{n}=\left(n^{(1)}, n^{(2)}, n^{(3)}\right)^{T}$ has boundary traction

$$
\mathbf{n} \cdot \mathcal{T}(\mathbf{u})=n^{(1)} A_{1} \nabla \mathbf{u}+n^{(2)} A_{2} \nabla \mathbf{u}+n^{(3)} A_{3} \nabla \mathbf{u} .
$$

A free surface boundary condition corresponds to $\mathbf{n} \cdot \mathcal{T}(\mathbf{u})=0$.

\subsection{Energy estimate}

For a box-shaped domain, $\Omega=\left\{0 \leq x^{(1)} \leq a^{(1)}, 0 \leq x^{(2)} \leq a^{(2)}, 0 \leq\right.$ $\left.x^{(3)} \leq a^{(3)}\right\}$, we define the $L_{2}$ scalar product of two real vector-valued functions $\mathbf{u}(\mathbf{x}) \in \Re^{3} \rightarrow \Re^{q}$ and $\mathbf{v}(\mathbf{x}) \in \Re^{3} \rightarrow \Re^{q}$ by

$$
(\mathbf{u}, \mathbf{v})_{2}=\int_{\Omega}\left(\sum_{l=1}^{q} u^{(l)} v^{(l)}\right) d x^{(1)} d x^{(2)} d x^{(3)} .
$$

An energy estimate for the solution of the elastic wave equation can be derived by analyzing the scalar product between $\mathbf{u}_{t}$ and (1),

$$
\left(\mathbf{u}_{t}, \rho \mathbf{u}_{t t}\right)_{2}=\left(\mathbf{u}_{t}, \mathbf{L u}\right)_{2}+\left(\mathbf{u}_{t}, \mathbf{F}\right)_{2} .
$$

From (7),

$$
\begin{aligned}
(\mathbf{v}, \mathbf{L u})_{2}=\left(\mathbf{v}, \partial_{1}\left(A_{1} \nabla \mathbf{u}\right)+\partial_{2}\left(A_{2} \nabla \mathbf{u}\right)+\partial_{3}\left(A_{3} \nabla \mathbf{u}\right)\right)_{2} \\
=:-S(\mathbf{v}, \mathbf{u})+B(\mathbf{v}, \mathbf{u}) .
\end{aligned}
$$

Here, $S$ and $B$ represent interior and boundary terms, respectively. After integration by parts, the interior term can be written

$$
\begin{aligned}
S(\mathbf{v}, \mathbf{u})= & \left(\partial_{1} \mathbf{v}, M^{11} \partial_{1} \mathbf{u}+M^{12} \partial_{2} \mathbf{u}+M^{13} \partial_{3} \mathbf{u}\right)_{2} \\
+ & \left(\partial_{2} \mathbf{v}, M^{21} \partial_{1} \mathbf{u}+M^{22} \partial_{2} \mathbf{u}+M^{23} \partial_{3} \mathbf{u}\right)_{2} \\
& +\left(\partial_{3} \mathbf{v}, M^{31} \partial_{1} \mathbf{u}+M^{32} \partial_{2} \mathbf{u}+M^{33} \partial_{3} \mathbf{u}\right)_{2}
\end{aligned}
$$

The definition of $M^{j k}$ in (6) gives, $\partial_{j} \mathbf{v}^{T} M^{j k} \partial_{k} \mathbf{u}=\partial_{j} \mathbf{v}^{T} P_{j}^{T} C P_{k} \partial_{k} \mathbf{u}=$ $\left(P_{j} \partial_{j} \mathbf{v}\right)^{T} C P_{k} \partial_{k} \mathbf{u}$, so that

$$
\begin{array}{r}
S(\mathbf{v}, \mathbf{u})=\sum_{j=1}^{3} \sum_{k=1}^{3}\left(P_{j} \partial_{j} \mathbf{v}, C P_{k} \partial_{k} \mathbf{u}\right)_{2}=\left(\sum_{j=1}^{3} P_{j} \partial_{j} \mathbf{v}, C \sum_{k=1}^{3} P_{k} \partial_{k} \mathbf{u}\right)_{2} \\
=\left(G_{s} \mathbf{v}, C G_{s} \mathbf{u}\right)_{2} .
\end{array}
$$


It follows from the positive definiteness of $C$ that $S$ is symmetric and positive semi-definite,

$$
S(\mathbf{v}, \mathbf{u})=S(\mathbf{u}, \mathbf{v}), \quad S(\mathbf{u}, \mathbf{u})=\left(G_{s} \mathbf{u}, C G_{s} \mathbf{u}\right)_{2} \geq 0 .
$$

Because $C$ is positive definite, the null-space of $S$ consists of functions $\mathbf{u}$ such that $G_{s} \mathbf{u}=0$. It is straightforward to show that there are six linearly independent functions that satisfy $G_{s} \mathbf{u}=0$, corresponding to solid body translations in the three coordinate directions and solid body rotations around the three coordinate axes.

The boundary term of (15) satisfies

$$
\begin{aligned}
B(\mathbf{v}, \mathbf{u})=\int_{x^{(2)}=0}^{a^{(2)}} & \int_{x^{(3)}=0}^{a^{(3)}}\left[\mathbf{v}^{T} A_{1} \nabla \mathbf{u}\right]_{x^{(1)}=0}^{a^{(1)}} d x^{(2)} d x^{(3)} \\
& +\int_{x^{(1)}=0}^{a^{(1)}} \int_{x^{(3)}=0}^{a^{(3)}}\left[\mathbf{v}^{T} A_{2} \nabla \mathbf{u}\right]_{x^{(2)}=0}^{a^{(2)}} d x^{(1)} d x^{(3)} \\
& +\int_{x^{(1)}=0}^{a^{(1)}} \int_{x^{(2)}=0}^{a^{(2)}}\left[\mathbf{v}^{T} A_{3} \nabla \mathbf{u}\right]_{x^{(3)}=0}^{a^{(3)}} d x^{(1)} d x^{(2)} .
\end{aligned}
$$

Obviously, $B(\mathbf{v}, \mathbf{u})=0$ if $\mathbf{v}$ satisfies homogeneous Dirichlet conditions. The first term on the right hand side of (18) is evaluated along the boundaries $x^{(1)}=0$ and $x^{(1)}=a^{(1)}$, respectively. Here the normal is $\mathbf{n}=(\mp 1,0,0)^{T}$, and $A_{1} \nabla \mathbf{u}$ equals the boundary traction. Hence, if a free surface condition is imposed along $x^{(1)}=0$ or $x^{(1)}=a^{(1)}$, we have $A_{1} \nabla \mathbf{u}=0$. The same argument applies to the second and third terms. Therefore, $B(\mathbf{v}, \mathbf{u})=0$ if $\mathbf{u}$ satisfies free surface conditions on all boundaries. In summary,

$$
B(\mathbf{v}, \mathbf{u})=0, \quad \text { if } \mathbf{v}=0, \text { or } \mathbf{n} \cdot \mathcal{T}(\mathbf{u})=0, \text { for } \mathbf{x} \in \partial \Omega .
$$

From (14) and (15) it follows that

$$
\frac{1}{2} \frac{d}{d t}\left(\left\|\sqrt{\rho} \mathbf{u}_{t}\right\|_{2}^{2}+S(\mathbf{u}, \mathbf{u})\right)=B\left(\mathbf{u}_{t}, \mathbf{u}\right)+\left(\mathbf{u}_{t}, \mathbf{F}\right)_{2} .
$$

The terms on the left hand side, $\left\|\sqrt{\rho} \mathbf{u}_{t}\right\|_{2}^{2}$ and $S(\mathbf{u}, \mathbf{u})$, represent the kinematic and potential energies, respectively. The boundary term $B\left(\mathbf{u}_{t}, \mathbf{u}\right)=0$ if $\mathbf{u}$ either satisfies homogeneous Dirichlet or free surface conditions, because if $\mathbf{u}=0$ on the boundary then also $\mathbf{u}_{t}=0$.

By integrating (20) in time,

$$
E(T)=E(0)+\int_{t=0}^{T}\left(\mathbf{u}_{t}, \mathbf{F}\right)_{2} d t, \quad E(t):=\frac{1}{2}\left(\left\|\sqrt{\rho} \mathbf{u}_{t}\right\|_{2}^{2}+\left(G_{s} \mathbf{u}, C\left(G_{s} \mathbf{u}\right)\right)_{2}\right),
$$


if $B\left(\mathbf{u}_{t}, \mathbf{u}\right)=0$. This shows that the solution of the elastic wave equation subject to homogeneous Dirichlet or free surface boundary conditions is a well-posed problem. In the absence of external forcing $(\mathbf{F}=0)$, we get $E(t)=E(0)$ for all $t>0$, i.e., the total energy of the solution is conserved.

\section{Generalization to curvilinear coordinates}

In this section we consider non-rectangular domains. Our presentation is essentially a generalization of the technique developed in [21].

Assume that there is a one-to-one mapping $\mathbf{x}=\mathbf{x}(\mathbf{r}):[0,1]^{3} \rightarrow \Omega \subset \Re^{3}$,

$$
\mathbf{x}(\mathbf{r})=\left(x^{(1)}(\mathbf{r}), x^{(2)}(\mathbf{r}), x^{(3)}(\mathbf{r})\right)^{T}, \quad \mathbf{r}=\left(r^{(1)}, r^{(2)}, r^{(3)}\right)^{T}, \quad 0 \leq r^{(k)} \leq 1,
$$

for $k=1,2,3$, from the unit cube in parameter space to the domain $\Omega$ in physical space. Denote partial differentiation with respect to the parameter coordinates by $\widetilde{\partial}_{k}=\partial / \partial r^{(k)}$. The relation between $\partial_{i}$ and $\widetilde{\partial}_{j}$ can be expressed in terms of the forward mapping function $\mathbf{x}=\mathbf{x}(\mathbf{r})$, or its inverse, $\mathbf{r}=\mathbf{r}(\mathbf{x}): \Omega \rightarrow[0,1]^{3}$, where $\mathbf{r}(\mathbf{x})=\left(r^{(1)}(\mathbf{x}), r^{(2)}(\mathbf{x}), r^{(3)}(\mathbf{x})\right)^{T}$. By the chain rule,

$$
\widetilde{\partial}_{q}=\sum_{p=1}^{3} \frac{\partial x^{(p)}}{\partial r^{(q)}} \partial_{p}, \quad \partial_{i}=\sum_{j=1}^{3} \xi_{i j} \widetilde{\partial}_{j}, \quad \xi_{i j}=\frac{\partial r^{(j)}}{\partial x^{(i)}} .
$$

for $q=1,2,3$ and $i=1,2,3$, respectively. The derivatives of the forward and inverse mapping functions define the covariant and contravariant base vectors,

$$
\mathbf{a}_{k}:=\widetilde{\partial}_{k} \mathbf{x}=\left(\begin{array}{c}
\partial x^{(1)} / \partial r^{(k)} \\
\partial x^{(2)} / \partial r^{(k)} \\
\partial x^{(3)} / \partial r^{(k)}
\end{array}\right), \mathbf{a}^{k}:=\nabla r^{(k)}=\left(\begin{array}{c}
\partial r^{(k)} / \partial x^{(1)} \\
\partial r^{(k)} / \partial x^{(2)} \\
\partial r^{(k)} / \partial x^{(3)}
\end{array}\right)=\left(\begin{array}{c}
\xi_{1 k} \\
\xi_{2 k} \\
\xi_{3 k}
\end{array}\right)
$$

for $k=1,2,3$, respectively. It is well-known that the contravariant base vectors can be expressed in terms of the covariant base vectors (see [22] for details),

$$
\mathbf{a}^{i}=\frac{1}{J}\left(\mathbf{a}_{j} \times \mathbf{a}_{k}\right), \quad(i, j, k) \text { cyclic, } \quad J=\operatorname{det}\left|\mathbf{a}_{1} \mathbf{a}_{2} \mathbf{a}_{3}\right| .
$$

Here, $J$ is the Jacobian of the forward mapping function. The above relation provides a convenient way of calculating the metric coefficients $\xi_{i j}$, which 

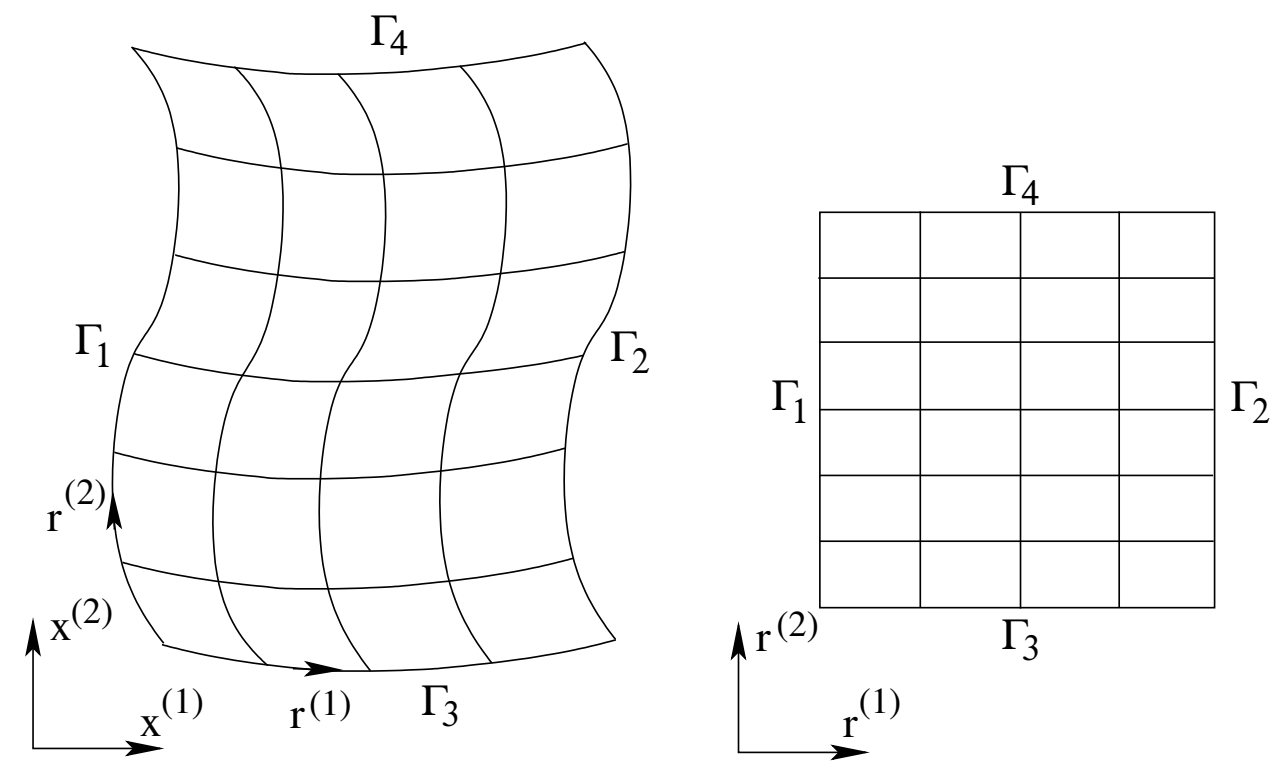

Figure 1: The mapping between physical (Cartesian) space (left) and parameter space (right) in the two-dimensional case. Here, boundary segments $\Gamma_{1}, \Gamma_{2}, \Gamma_{3}$, and $\Gamma_{4}$ are mapped to to $r^{(1)}=0, r^{(1)}=1, r^{(2)}=0$, and $r^{(2)}=1$, respectively.

are needed in the curvilinear formulation of the elastic wave equation. In the following we assume that the mapping is non-singular, with $0<J<\infty$.

In Cartesian coordinates (left side of Figure 1), the elastic wave equation takes the form (1)-(2). In curvilinear coordinates, it is natural to formulate the elastic wave equation as

$$
\rho J \frac{\partial^{2} \mathbf{u}}{\partial t^{2}}=J \mathbf{L u}+J \mathbf{F}, \quad \mathbf{r} \in[0,1]^{3}, \quad t \geq 0 .
$$

We introduce the curvilinear mapping into (4) to obtain

$$
G_{s} \mathbf{u}=\sum_{j=1}^{3} P_{j} \partial_{j} \mathbf{u}=\sum_{j=1}^{3} P_{j} \sum_{k=1}^{3} \xi_{j k} \widetilde{\partial}_{k} \mathbf{u}=\sum_{k=1}^{3} \widetilde{P}_{k} \widetilde{\partial}_{k} \mathbf{u}
$$

where

$$
\widetilde{P}_{k}=\sum_{j=1}^{3} \xi_{j k} P_{j}
$$

This definition gives the divergence of the stress tensor in curvilinear coor- 
dinates,

$$
\begin{aligned}
\mathbf{L u}=G_{s}^{T} C G_{s} \mathbf{u}=\sum_{j=1}^{3} \widetilde{P}_{j}^{T} \widetilde{\partial}_{j}\left(C \sum_{k=1}^{3} \widetilde{P}_{k} \widetilde{\partial}_{k} \mathbf{u}\right) & \\
& =\frac{1}{J} \sum_{j=1}^{3} \sum_{k=1}^{3} \widetilde{\partial}_{j}\left(J \widetilde{P}_{j}^{T} C \widetilde{P}_{k} \widetilde{\partial}_{k} \mathbf{u}\right) .
\end{aligned}
$$

Here we used the metric identities

$$
\tilde{\partial}_{1}\left(J \xi_{k 1}\right)+\tilde{\partial}_{2}\left(J \xi_{k 2}\right)+\tilde{\partial}_{3}\left(J \xi_{k 3}\right)=0, \quad k=1,2,3,
$$

which follow from (23) (also see [22]). This identity allows the term $J \widetilde{P}_{j}^{T}$ to be moved inside the differentiation $\widetilde{\partial}_{j}$ on the right hand side of (25). We define the matrices

$$
N^{j k}=J \widetilde{P}_{j}^{T} C \widetilde{P}_{k},
$$

and rewrite $(25)$ on the same form as $(7)-(10)$,

$$
J \mathbf{L u}=\tilde{\partial}_{1}\left(\widetilde{A}_{1} \widetilde{\nabla} \mathbf{u}\right)+\tilde{\partial}_{2}\left(\widetilde{A}_{2} \widetilde{\nabla} \mathbf{u}\right)+\tilde{\partial}_{3}\left(\widetilde{A}_{3} \widetilde{\nabla} \mathbf{u}\right),
$$

where

$$
\begin{aligned}
& \widetilde{A}_{1} \widetilde{\nabla} \mathbf{u}:=N^{11} \tilde{\partial}_{1} \mathbf{u}+N^{12} \tilde{\partial}_{2} \mathbf{u}+N^{13} \tilde{\partial}_{3} \mathbf{u}, \\
& \widetilde{A}_{2} \widetilde{\nabla} \mathbf{u}:=N^{21} \tilde{\partial}_{1} \mathbf{u}+N^{22} \tilde{\partial}_{2} \mathbf{u}+N^{23} \tilde{\partial}_{3} \mathbf{u}, \\
& \widetilde{A}_{3} \widetilde{\nabla} \mathbf{u}:=N^{31} \tilde{\partial}_{1} \mathbf{u}+N^{32} \tilde{\partial}_{2} \mathbf{u}+N^{33} \tilde{\partial}_{3} \mathbf{u}
\end{aligned}
$$

The definition (27) makes it straightforward to verify that the matrices $N^{j k}$ have the same properties as the matrices $M^{j k}$, i.e., $N^{11}, N^{22}$, and $N^{33}$ are positive definite and $N^{k j}=\left(N^{j k}\right)^{T}$.

\subsection{Boundary conditions}

To transform a free surface boundary condition to curvilinear coordinates, we first note that the boundary normal can be represented by the metric derivatives. For example, along $r^{(1)}=0$ or $r^{(1)}=1$, the outwardly directed unit normal satisfies

$$
\mathbf{n}:=\left(\begin{array}{l}
n^{(1)} \\
n^{(2)} \\
n^{(3)}
\end{array}\right)=\mp \frac{\nabla r^{(1)}}{\left|\nabla r^{(1)}\right|}=\frac{\mp 1}{\sqrt{\left(\xi_{11}\right)^{2}+\left(\xi_{21}\right)^{2}+\left(\xi_{31}\right)^{2}}}\left(\begin{array}{l}
\xi_{11} \\
\xi_{21} \\
\xi_{31}
\end{array}\right)
$$


for $r^{(1)}=0$, or $r^{(1)}=1$, where the minus sign applies to $r^{(1)}=0$. The components of the stress tensor are given by (11). Using (21), the gradient of $\mathbf{u}$ in (8)-(10) can be expressed in terms of derivatives with respect to the parameter coordinates. After some straightforward algebra,

$$
\mathbf{n} \cdot \mathcal{T}=\frac{\mp 1}{J \mid \nabla r^{(1) \mid}}\left(N^{11} \tilde{\partial}_{1} \mathbf{u}+N^{12} \tilde{\partial}_{2} \mathbf{u}+N^{13} \tilde{\partial}_{3} \mathbf{u}\right), \quad r^{(1)}=0,1 .
$$

The definition of $\widetilde{A_{1}} \widetilde{\nabla} \mathbf{u}$ in (29) finally gives

$$
\mathbf{n} \cdot \mathcal{T}=\frac{\mp 1}{J\left|\nabla r^{(1)}\right|} \widetilde{A}_{1} \widetilde{\nabla} \mathbf{u}, \quad r^{(1)}=0,1
$$

In a similar way,

$$
\begin{aligned}
& \mathbf{n} \cdot \mathcal{T}=\frac{\mp 1}{J\left|\nabla r^{(2)}\right|} \widetilde{A}_{2} \widetilde{\nabla} \mathbf{u}, \quad r^{(2)}=0,1, \\
& \mathbf{n} \cdot \mathcal{T}=\frac{\mp 1}{J\left|\nabla r^{(3)}\right|} \widetilde{A}_{3} \widetilde{\nabla} \mathbf{u}, \quad r^{(3)}=0,1 .
\end{aligned}
$$

\subsection{Energy estimate}

By using (24)-(28), the elastic wave equation in curvilinear coordinates can be written

$$
\rho \frac{\partial^{2} \mathbf{u}}{\partial t^{2}}=\frac{1}{J}\left[\tilde{\partial}_{1}\left(\widetilde{A}_{1} \widetilde{\nabla} \mathbf{u}\right)+\tilde{\partial}_{2}\left(\widetilde{A}_{2} \widetilde{\nabla} \mathbf{u}\right)+\tilde{\partial}_{3}\left(\widetilde{A}_{3} \widetilde{\nabla} \mathbf{u}\right)\right]+\mathbf{F},
$$

for $\mathbf{r} \in[0,1]^{3}$ and $t \geq 0$. In curvilinear coordinates, the volume element in an integral is scaled by $J$, and the $L_{2}$ scalar product (13) becomes

$$
(\mathbf{u}, \mathbf{v})_{2}=\int_{\mathbf{r} \in[0,1]^{3}}\left(\sum_{l=1}^{q} u^{(l)} v^{(l)}\right) J d r^{(1)} d r^{(2)} d r^{(3)} .
$$

An energy estimate can be derived in the same way as in the Cartesian case, because the factor $J$ in the scalar product cancels the $1 / J$ on the right hand side of (36). Partial integration gives a spatial decomposition of the form (15). The only difference is that the matrices $M^{j k}$, which describe the material properties in the Cartesian case, are replaced by the matrices $N^{j k}$, which describe the corresponding material properties in parameter space $\mathbf{r} \in[0,1]^{3}$. Since this is the only difference, the interior term $S(\mathbf{v}, \mathbf{u})$ is symmetric and positive semi-definite also in curvilinear coordinates. In curvilinear coordinates, free surface conditions take the form (33)-(35), and Dirichlet boundary conditions are transformed trivially. Hence, the boundary term $B\left(\mathbf{u}_{t}, \mathbf{u}\right)=0$ if $\mathbf{u}$ either satisfies free surface or homogeneous Dirichlet conditions. Under such boundary conditions, the elastic wave equation in curvilinear coordinates is a well-posed problem. 


\subsection{Isotropic elastic material in curvilinear coordinates}

In the special case of an isotropic elastic material, we have

$$
\begin{gathered}
M_{i s o}^{11}=\left(\begin{array}{ccc}
2 \mu+\lambda & 0 & 0 \\
0 & \mu & 0 \\
0 & 0 & \mu
\end{array}\right), \quad M_{i s o}^{12}=\left(\begin{array}{ccc}
0 & \lambda & 0 \\
\mu & 0 & 0 \\
0 & 0 & 0
\end{array}\right), \quad M_{i s o}^{13}=\left(\begin{array}{lll}
0 & 0 & \lambda \\
0 & 0 & 0 \\
\mu & 0 & 0
\end{array}\right), \\
M_{i s o}^{21}=\left(M_{i s o}^{12}\right)^{T}, \quad M_{i s o}^{22}=\left(\begin{array}{ccc}
\mu & 0 & 0 \\
0 & 2 \mu+\lambda & 0 \\
0 & 0 & \mu
\end{array}\right), \quad M_{i s o}^{23}=\left(\begin{array}{lll}
0 & 0 & 0 \\
0 & 0 & \lambda \\
0 & \mu & 0
\end{array}\right), \\
M_{i s o}^{31}=\left(M_{i s o}^{13}\right)^{T}, \quad M_{i s o}^{32}=\left(M_{i s o}^{23}\right)^{T}, \quad M_{i s o}^{33}=\left(\begin{array}{ccc}
\mu & 0 & 0 \\
0 & \mu & 0 \\
0 & 0 & 2 \mu+\lambda
\end{array}\right) .
\end{gathered}
$$

Here, $\lambda$ and $\mu$ are the first and second Lamé parameters, respectively.

In curvilinear coordinates, the corresponding material properties are described by the matrices $N^{i j}$, defined in (27). For example, $N^{11}$ satisfies

$N_{\text {iso }}^{11}=J \mu\left(\xi_{11}^{2}+\xi_{21}^{2}+\xi_{31}^{2}\right)\left(\begin{array}{ccc}1 & 0 & 0 \\ 0 & 1 & 0 \\ 0 & 0 & 1\end{array}\right)+J(\lambda+\mu)\left(\begin{array}{ccc}\xi_{11}^{2} & \xi_{11} \xi_{21} & \xi_{11} \xi_{31} \\ \xi_{11} \xi_{21} & \xi_{21}^{2} & \xi_{21} \xi_{31} \\ \xi_{11} \xi_{31} & \xi_{21} \xi_{31} & \xi_{31}^{2}\end{array}\right)$.

The remaining $N^{i j}$ are of a similar form. For a general curvilinear mapping, note that the transformed matrices do not have any zero elements. Hence, because of the coordinate mapping, the isotropic material has anisotropic properties in curvilinear parameter space.

\section{Discretization of the elastic wave equation}

To conserve space we only describe the discretization in curvilinear coordinates. The Cartesian case follows by using the semi-trivial mapping function $x^{(k)}\left(r^{(k)}\right)=a^{(k)} r^{(k)}, k=1,2,3$, where $a^{(k)}$ are constants.

We consider the elastic wave equation in curvilinear coordinates (24) where $J \mathbf{L u}$ is given by (28). We re-order the terms of the spatial operator such that

$$
\begin{aligned}
\mathbf{L u}=\frac{1}{J} & {\left[\tilde{\partial}_{1}\left(N^{11} \tilde{\partial}_{1} \mathbf{u}\right)+\tilde{\partial}_{2}\left(N^{22} \tilde{\partial}_{2} \mathbf{u}\right)+\tilde{\partial}_{3}\left(N^{33} \tilde{\partial}_{3} \mathbf{u}\right)+\tilde{\partial}_{1}\left(N^{12} \tilde{\partial}_{2} \mathbf{u}\right)+\tilde{\partial}_{1}\left(N^{13} \tilde{\partial}_{3} \mathbf{u}\right)\right.} \\
& \left.+\tilde{\partial}_{2}\left(N^{21} \tilde{\partial}_{1} \mathbf{u}\right)+\tilde{\partial}_{2}\left(N^{23} \tilde{\partial}_{3} \mathbf{u}\right)+\tilde{\partial}_{3}\left(N^{31} \tilde{\partial}_{1} \mathbf{u}\right)+\tilde{\partial}_{3}\left(N^{32} \tilde{\partial}_{2} \mathbf{u}\right)\right] \cdot
\end{aligned}
$$


A uniform grid, $r_{i}^{(1)}=(i-1) h_{1}, i=0, \ldots, n_{1}+1, r_{j}^{(2)}=(j-1) h_{2}, j=$ $0, \ldots, n_{2}+1$, and $r_{k}^{(3)}=(k-1) h_{3}, k=0, \ldots, n_{3}+1$ discretizes the domain in parameter space. Here, the grid spacings are $h_{1}=1 /\left(n_{1}-1\right), h_{2}=1 /\left(n_{2}-1\right)$, and $h_{3}=1 /\left(n_{3}-1\right)$. The points outside the domain are ghost points, which are used to impose the boundary conditions.

Before presenting our spatial discretization of (38), we first review some well-known properties of summation-by-parts (SBP) finite difference operators in a one-dimensional setting.

\subsection{SBP finite difference operators}

Assume that a one-dimensional domain is discretized by the uniform grid $x_{i}=(i-1) h$ for $i=0, \ldots, n+1$, where the domain boundaries are at $i=1$ and $i=n$. Let $u_{i}$ be a real-valued function defined on the grid. We say that the difference operator $D$, approximating $d / d x$, satisfies the property of SBP if

$$
(u, D v)_{h 1}=-(D u, v)_{h 1}-u_{1} v_{1}+u_{n} v_{n}
$$

in a scalar product,

$$
(u, v)_{h 1}=h \sum_{i=1}^{n} \omega_{i} u_{i} v_{i}, \quad 0<\omega_{i}<\infty,
$$

where $\omega_{i}$ are the weights of the scalar product. The grid function $D u_{i}$ is defined at all points $i=1, \ldots, n$. Away from the boundaries, $D u_{i}$ equals a centered difference operator. In order to satisfy (39), the coefficients in $D$ are modified at a few points near each boundary. When using a scalar product of the form (40), it is well known that (39) can only be satisfied if the order of the truncation error in $D u$ is reduced by a factor of two at a few points near each boundary. It is possible to improve the truncation error near the boundary by using so-called full norm SBP operators [23]. However, these operators can lead to instabilities with variable coefficients and will not be used here.

In the following our presentation assumes a scalar product of the form (40).

SBP operators of order $p$ away from the boundaries and order $p / 2$ near the boundaries, for $p=2,4,6,8$, are well-documented in the literature, see e.g. [23]. It is theoretically possible to derive even higher order accurate SBP operators, but the stencils become very wide and the coefficients depend on a number of parameters which can be difficult to determine.

Second derivative terms of the type $\left.\left(a(x) u_{x}\right)_{x}\right)$ appear in the elastic wave equation. Here $a(x)$ is a known function that describes a material 
property such as the shear modulus. These terms could be approximated by applying $D$ twice. However, this approach leads to difficulties with odd-even modes, meaning that the null space of $D(a D u)_{j}$ contains highly oscillatory grid functions. Furthermore, because of the boundary modification, the truncation error of $D$ is not smooth near the boundary, leading to additional loss of accuracy during the second application of $D$.

In [11], we constructed a difference operator $G(a) u$ approximating $\left(a u_{x}\right)_{x}$, which does not have problems with odd-even modes. This operator satisfies the SBP identity

$$
(v, G(a) u)_{h 1}=-(D v, a D u)_{h 1}+(v, P(a) u)_{h r 1}-v_{1} a_{1} S^{b} u_{1}+v_{n} a_{n} S^{b} u_{n} .
$$

Here, $S^{b}$ is a difference operator approximating the first derivative on the boundary, to full order of accuracy $(p)$. The operator is of the form $S^{b} u_{1}=$ $(1 / h) \sum_{k=0}^{m-1} s_{k} u_{k}$, where $s_{k}$ are constant stencil coefficients. Note that $S^{b} u_{1}$ makes use of the ghost point value $u_{0}$. The operator $S^{b} u_{n}$ is similar, and uses the ghost point value $u_{n+1}$. The positive semi-definite operator $P(a)$ is small and non-zero for odd-even modes. Note that the scalar product $(v, u)_{h r 1}$ is weighted differently than $(u, v)_{h 1}$, see [11] for details.

The operator $G(a)$ derived in [11] is fourth order accurate in the interior and second order near the boundary. It is designed to be compatible with the operator $D$ in (41), which is the standard SBP $4^{\text {th }} / 2^{\text {nd }}$ order accurate approximation of the first derivative. Because the elastic wave equation is solved in second order formulation, two orders of accuracy are gained in the solution, which becomes fourth order accurate in maximum norm. Extensions to even higher order is possible, but not pursued here.

We remark that there is a related SBP method for discretizing $\left(a u_{x}\right)_{x}$, which does not use ghost points and instead enforces the boundary conditions by a penalty techniques, see e.g. $[14,15,16]$.

The spatial operator of the elastic wave equation (38) consists of terms such as $\tilde{\partial}_{i}\left(N^{i j} \tilde{\partial}_{j} \mathbf{u}\right)$. Here $N^{i j}$ is a $3 \times 3$ matrix with elements $n_{p q}^{i j}$. When $i=j$, the term is approximated by $G\left(N^{i i}\right) \mathbf{u}$, defined as

$$
G\left(N^{i i}\right) \mathbf{u}:=\left(\begin{array}{c}
\left(G\left(N^{i i}\right) \mathbf{u}\right)_{1} \\
\left(G\left(N^{i i}\right) \mathbf{u}\right)_{2} \\
\left(G\left(N^{i i}\right) \mathbf{u}\right)_{3}
\end{array}\right), \quad\left(G\left(N^{i i}\right) \mathbf{u}\right)_{p}=\sum_{q=1}^{3} G\left(n_{p q}^{i i}\right) u^{(q)},
$$

for $p=1,2,3$, where $G\left(n_{p q}^{i i}\right) u^{(q)}$ is the scalar difference operator described above. 
For vector valued grid functions, we approximate $\tilde{\partial}_{j} \mathbf{u}$ using the operator $D_{j} \mathbf{u}$, which is defined component-wise. This operator is used to approximate $\tilde{\partial}_{i}\left(N^{i j} \tilde{\partial}_{j} \mathbf{u}\right)$ when $i \neq j$.

The vector version of the SBP identities (39) and (41) are

$$
\begin{aligned}
(\mathbf{u}, D \mathbf{v})_{h 1} & =-(D \mathbf{u}, \mathbf{v})_{h 1}-\mathbf{u}_{1}^{T} \mathbf{v}_{1}+\mathbf{u}_{n}^{T} \mathbf{v}_{n} \\
(\mathbf{v}, G(N) \mathbf{u})_{h 1}=-(D \mathbf{v}, & N D \mathbf{u})_{h 1}+(\mathbf{v}, P(N) \mathbf{u})_{h r 1} \\
& -\mathbf{v}_{1}^{T} N_{1} S^{b} \mathbf{u}_{1}+\mathbf{v}_{n}^{T} N_{n} S^{b} \mathbf{u}_{n}
\end{aligned}
$$

as can be seen by component-wise application of the corresponding scalar identities.

\subsection{Spatial discretization}

The spatial operator (38) is discretized as

$$
\begin{aligned}
\mathbf{L}_{h} \mathbf{u}_{i, j, k}=\frac{1}{J_{i, j, k}}[ & G_{1}\left(N^{11}\right) \mathbf{u}_{i, j, k}+G_{2}\left(N^{22}\right) \mathbf{u}_{i, j, k}+G_{3}\left(N^{33}\right) \mathbf{u}_{i, j, k} \\
& +D_{1}\left(N^{12} D_{2} \mathbf{u}_{i, j, k}\right)+D_{1}\left(N^{13} D_{3} \mathbf{u}_{i, j, k}\right) \\
& +D_{2}\left(N^{21} D_{1} \mathbf{u}_{i, j, k}\right)+D_{2}\left(N^{23} D_{3} \mathbf{u}_{i, j, k}\right) \\
& \left.+D_{3}\left(N^{31} D_{1} \mathbf{u}_{i, j, k}\right)+D_{3}\left(N^{32} D_{2} \mathbf{u}_{i, j, k}\right)\right]
\end{aligned}
$$

for $i=1, \ldots, n_{1}, j=1, \ldots, n_{2}$, and $k=1, \ldots, n_{3}$. Here, $D_{m}$ is the standard SBP finite difference operator acting in one of the curvilinear coordinate directions $m=1,2,3$. Similarly, $G_{m}(N)$ denotes the second derivative operator (42) acting in direction $m$. The discrete scalar product is defined by

$$
(\mathbf{u}, \mathbf{v})_{h}=h_{1} h_{2} h_{3} \sum_{i=1}^{n_{1}} \sum_{j=1}^{n_{2}} \sum_{k=1}^{n_{3}} \omega_{i}^{(1)} \omega_{j}^{(2)} \omega_{k}^{(3)} J_{i, j, k} \mathbf{u}_{i, j, k}^{T} \mathbf{v}_{i, j, k} .
$$

The matrices $N^{i j}$ are defined by (27). If analytical expressions for the derivatives of the coordinate mapping are available, they simply need to be evaluated at the grid points. However, if they are not available, they can instead be approximated by sufficiently accurate numerical differentiation,

$$
\left.\frac{\partial x^{(q)}}{\partial r^{(p)}}\right|_{i, j, k} \approx D_{p} x_{i, j, k}^{(q)}, \quad p=1,2,3, \quad q=1,2,3 .
$$

In this case, the covariant base vectors follow from $(22)$, with $\partial x^{(q)} / \partial r^{(p)}$ replaced by $D_{p} x^{(q)}$. The metric coefficients $\xi_{p q}$ and the discretized Jacobian, $J_{i, j, k}$ are given by formula (23). 
Remark 1. The difference operators can be evaluated undivided, i.e., the grid sizes, $h_{1}, h_{2}$, and $h_{3}$ can be set to one when evaluating $D \mathbf{u}$ and $G(N) \mathbf{u}$, if also the metric difference approximations $D_{p} x_{i, j, k}^{(q)}$ are treated in the same way. This is because the grid sizes $\left(h_{i}\right)$ cancel in the expression for $\mathbf{L}_{h} \mathbf{u}$.

Remark 2. The metric identities (26) are in general not satisfied exactly when the metric derivatives are approximated by the difference formula (46). When first order hyperbolic systems are discretized on curvilinear grids, not satisfying the corresponding metric identities implies that the numerical approximation of derivatives of constant functions can be non-zero. This, in turn, can lead to spurious numerical effects when trying to preserve a constant state over long times, unless special free stream preserving metric discretizations are used [24, 25, 26]. Because we here solve a hyperbolic system in second order formulation, $\mathbf{L}_{h} \mathbf{u}$ is identically zero for constant $\mathbf{u}$, also when the difference formula (46) is used to approximate the metric derivatives. In practical calculations we have always found that these simpler difference formulas are adequate.

The discrete analog of (15) is

$$
\left(\mathbf{v}, \mathbf{L}_{h} \mathbf{u}\right)_{h}=-S_{h}(\mathbf{v}, \mathbf{u})+B_{h}(\mathbf{v}, \mathbf{u}),
$$

where $S_{h}$ is symmetric and positive semi-definite, and $B_{h}$ contains boundary terms. To verify this equality we may proceed in the following way. We first multiply (45) by $\omega^{(1)} \omega^{(2)} \omega^{(3)} J \mathbf{v}^{T}$ from the left, sum over all grid points, and finally apply the one-dimensional identities (43) and (44) along each curvilinear coordinate direction. This results in

$$
\begin{gathered}
\left(\mathbf{v}, \mathbf{L}_{h} \mathbf{u}\right)_{h}=-\left(D_{1} \mathbf{v}, N^{11} D_{1} \mathbf{u}\right)_{h}-\left(D_{1} \mathbf{v}, N^{12} D_{2} \mathbf{u}\right)_{h}-\left(D_{1} \mathbf{v}, N^{13} D_{3} \mathbf{u}\right)_{h} \\
-\left(D_{2} \mathbf{v}, N^{21} D_{1} \mathbf{u}\right)_{h}-\left(D_{2} \mathbf{v}, N^{22} D_{2} \mathbf{u}\right)_{h}-\left(D_{2} \mathbf{v}, N^{23} D_{3} \mathbf{u}\right)_{h} \\
-\left(D_{3} \mathbf{v}, N^{31} D_{3} \mathbf{u}\right)_{h}-\left(D_{3} \mathbf{v}, N^{32} D_{2} \mathbf{u}\right)_{h}-\left(D_{3} \mathbf{v}, N^{33} D_{3} \mathbf{u}\right)_{h} \\
-\left(\mathbf{v}, P_{1}\left(N^{11}\right) \mathbf{u}\right)_{h r}-\left(\mathbf{v}, P_{2}\left(N^{22}\right) \mathbf{u}\right)_{h r}-\left(\mathbf{v}, P_{3}\left(N^{33}\right) \mathbf{u}\right)_{h r}+B_{h}(\mathbf{v}, \mathbf{u}) .
\end{gathered}
$$

All terms on the right hand side, except the last one, define $S_{h}(\mathbf{v}, \mathbf{u})$. The 
boundary term is given by

$$
\begin{aligned}
B_{h}(\mathbf{v}, \mathbf{u})= & h_{2} h_{3} \sum_{j=1}^{n_{2}} \sum_{k=1}^{n_{3}} \omega_{j}^{(2)} \omega_{k}^{(3)}\left[\mathbf{v}_{i, j, k}^{T} \widetilde{A}_{1, h} \widetilde{\nabla}_{h} \mathbf{u}_{i, j, k}\right]_{i=1}^{i=n_{1}} \\
+ & h_{1} h_{3} \sum_{i=1}^{n_{1}} \sum_{k=1}^{n_{3}} \omega_{i}^{(1)} \omega_{k}^{(3)}\left[\mathbf{v}_{i, j, k}^{T} \widetilde{A}_{2, h} \widetilde{\nabla}_{h} \mathbf{u}_{i, j, k}\right]_{j=1}^{j=n_{2}} \\
& +h_{1} h_{2} \sum_{i=1}^{n_{1}} \sum_{j=1}^{n_{2}} \omega_{i}^{(1)} \omega_{j}^{(2)}\left[\mathbf{v}_{i, j, k}^{T} \widetilde{A}_{3, h} \widetilde{\nabla}_{h} \mathbf{u}_{i, j, k}\right]_{k=1}^{k=n_{3}},
\end{aligned}
$$

with

$$
\begin{aligned}
\widetilde{A}_{1, h} \widetilde{\nabla}_{h} \mathbf{u} & =N^{11} S_{1}^{b} \mathbf{u}+N^{12} D_{2} \mathbf{u}+N^{13} D_{3} \mathbf{u}, \\
\widetilde{A}_{2, h} \widetilde{\nabla}_{h} \mathbf{u} & =N^{21} D_{1} \mathbf{u}+N^{22} S_{2}^{b} \mathbf{u}+N^{23} D_{3} \mathbf{u}, \\
\widetilde{A}_{3, h} \widetilde{\nabla}_{h} \mathbf{u} & =N^{31} D_{1} \mathbf{u}+N^{32} D_{2} \mathbf{u}+N^{33} S_{3}^{b} \mathbf{u} .
\end{aligned}
$$

Energy conserving boundary conditions, i.e., boundary conditions that make $B_{h}(\mathbf{v}, \mathbf{u})=0$, can be imposed either by a homogeneous Dirichlet condition,

$$
\mathbf{v}_{i, j, k}=\mathbf{0}, \quad(i, j, k) \text { on the boundary, }
$$

or by a free-surface condition,

$$
\widetilde{A}_{q, h} \widetilde{\nabla}_{h} \mathbf{u}_{i, j, k}=\mathbf{0}, \quad(i, j, k) \text { on the boundary. }
$$

Here, $q=1,2$, or 3 depending on which side is being considered. For example, on the boundary $k=1$, we have

$N_{i, j, 1}^{31} D_{1} \mathbf{u}_{i, j, 1}+N_{i, j, 1}^{32} D_{2} \mathbf{u}_{i, j, 1}+N_{i, j, 1}^{33} S_{3}^{b} \mathbf{u}_{i, j, 1}=0, \quad i=1, \ldots, n_{1}, \quad j=1, \ldots, n_{2}$.

This constitutes a system of three equations for the three unknowns $\mathbf{u}_{i, j, 0}$,

$$
s_{0} N_{i, j, 1}^{33} \mathbf{u}_{i, j, 0}=-N_{i, j, 1}^{33} \sum_{k=1}^{m-1} s_{k} \mathbf{u}_{i, j, k}-N_{i, j, 1}^{31} D_{1} \mathbf{u}_{i, j, 1}-N_{i, j, 1}^{32} D_{2} \mathbf{u}_{i, j, 1} .
$$

Because $N_{i, j, 1}^{33}$ is positive definite and $s_{0} \neq 0$, this system always has a unique solution. Note that the system only couples the three ghost point values $\mathbf{u}_{i, j, 0}$, for each $(i, j)$. There is no coupling along the boundary.

By comparing (49) with (32), we note that the former is an approximation of the scaled boundary traction, where the scaling factor $J\left|\nabla r^{(1)}\right|$ is the 
surface measure. To make this obvious, we can write the first sum of the right hand side of (48) as (omitting the factor $h_{2} h_{3}$ )

$$
\begin{aligned}
\sum_{j=1}^{n_{2}} \sum_{k=1}^{n_{3}} \omega_{j}^{(2)} \omega_{k}^{(3)}[ & \left.J_{i, j, k}\left|\nabla r^{(1)}\right| \mathbf{v}_{i, j, k}^{T} \frac{1}{J_{i, j, k}\left|\nabla r^{(1)}\right|} \widetilde{A}_{1, h} \widetilde{\nabla}_{h} \mathbf{u}_{i, j, k}\right]_{i=1}^{i=n_{1}}= \\
& \sum_{j=1}^{n_{2}} \sum_{k=1}^{n_{3}} \omega_{j}^{(2)} \omega_{k}^{(3)}\left[J_{i, j, k}\left|\nabla r^{(1)}\right| \mathbf{v}_{i, j, k}^{T}\left(\mathbf{n} \cdot \mathcal{T}_{h}\right)_{i, j, k}\right]_{i=1}^{i=n_{1}}
\end{aligned}
$$

and similarly for the other two sums. Here, $\mathbf{n} \cdot \mathcal{T}_{h}=\frac{1}{J\left|\nabla r^{(1)}\right|} \widetilde{A}_{1, h} \widetilde{\nabla}_{h} \mathbf{u}$ is the discretization of the boundary traction (32).

Finally, when energy conserving boundary conditions are imposed, the semi-discrete energy estimate

$$
\frac{1}{2} \frac{d}{d t}\left(\left(\rho \mathbf{u}_{t}, \mathbf{u}_{t}\right)_{h}+S_{h}(\mathbf{u}, \mathbf{u})\right)=\left(\mathbf{u}_{t}, \mathbf{F}\right)_{h}
$$

follows in the same way as the corresponding estimate for the continuous problem. This leads to stability if the energy, $\left(\rho \mathbf{u}_{t}, \mathbf{u}_{t}\right)_{h}+S_{h}(\mathbf{u}, \mathbf{u})$, is positive. Our SBP discretization has the property that $S_{h}$ is positive semidefinite with a null space that is a discretized approximation of the null space of the continuous operator, i.e., solid body translations and rotations. For example, the odd-even modes, $u_{j}=(-1)^{j}$ are not in the null space of $S_{h}$ because of the terms $\left(\mathbf{u}, P_{j}\left(N^{j j}\right) \mathbf{u}\right)_{h r}$, which are positive for such grid functions [11]. Also note that solid body translations and rotations are not possible if $\mathbf{u}$ satisfies homogeneous Dirichlet conditions on at least part of the boundary. In this case $S_{h}(\mathbf{u}, \mathbf{u})$ becomes positive definite and the SBP discretization is stable.

\subsection{Time discretization}

The equations are advanced in time with an explicit time integration method. As with all explicit time stepping methods, the time step must not exceed the CFL stability limit. With a Newmark time stepping scheme,

$$
\frac{\mathbf{u}^{n+1}-2 \mathbf{u}^{n}+\mathbf{u}^{n-1}}{\Delta_{t}^{2}}=\mathbf{L}_{h} \mathbf{u}^{n}+\mathbf{F}^{n}, \quad n=0,1, \ldots,
$$

the expression for the CFL time step limit is

$$
\frac{\Delta_{t}^{2} \zeta}{4} \leq 1
$$


The spectral radius $\zeta=\max _{\mathbf{u} \neq \mathbf{0}} S_{h}(\mathbf{u}, \mathbf{u}) /(\mathbf{u}, \rho \mathbf{u})_{h}$ is difficult to compute for a general heterogeneous material, even when the material is isotropic. As an approximation we consider the elastic wave equations in a homogeneous material with periodic boundary conditions. A von Neumann analysis of the Fourier transformed problem, in the case of second order accuracy, shows that the spectral radius in an isotropic elastic material is well approximated by

$$
\zeta \approx \frac{4}{h^{2}} \frac{2 \mu+\lambda+\mu+\mu}{\rho}=\frac{4}{h^{2}}\left(c_{p}^{2}+2 c_{s}^{2}\right) .
$$

Here, $c_{p}$ and $c_{s}$ are the longitudinal and transverse phase velocities, respectively.

In a general anisotropic material, the square of the phase velocity, $c^{2}$, is an eigenvalue of the Christoffel equation,

$$
c^{2} \mathbf{r}=\frac{1}{\rho} \sum_{j=1}^{3} \sum_{k=1}^{3} n_{j} n_{k} M^{j k} \mathbf{r} .
$$

In general, the phase velocity depends on the direction of wave propagation $\mathbf{n}=\left(n_{1}, n_{2}, n_{3}\right)^{T},|\mathbf{n}|=1$. In the isotropic case, the three eigenvalues of the Christoffel equation are $\left[c_{p}^{2}, c_{s}^{2}, c_{s}^{2}\right]$, independently of the direction $\mathbf{n}$. Since $h^{2} \zeta / 4$ can be approximated by the sum of the three eigenvalues of the Christoffel equation in the isotropic case, it is reasonable to assume that the sum of the eigenvalues of (57) would also be a good approximation of $h^{2} \zeta / 4$, in the anisotropic case. The sum of the eigenvalues of a matrix equals the trace of the matrix, i.e., the sum of its diagonal elements. Hence, the sum of the eigenvalues in the direction $\mathbf{n}=\left(n_{1}, n_{2}, n_{3}\right)^{T}$ is given by

$$
\frac{1}{\rho} \operatorname{Tr}\left(\sum_{j=1}^{3} \sum_{k=1}^{3} n_{j} n_{k} M^{j k}\right)=\frac{1}{\rho} \sum_{j=1}^{3} \sum_{k=1}^{3} n_{j} n_{k} \operatorname{Tr}\left(M^{j k}\right),
$$

where $\operatorname{Tr}(M)$ denotes the trace of $M$. The expression (58) is a quadratic form, whose maximum over all directions $\mathbf{n}$ equals the maximum eigenvalue, $\kappa_{\max }=\max \kappa$, defined by

$$
\operatorname{det}(T-\kappa I)=0, \quad T=\frac{1}{\rho}\left(\begin{array}{lll}
\operatorname{Tr}\left(M^{11}\right) & \operatorname{Tr}\left(M^{12}\right) & \operatorname{Tr}\left(M^{13}\right) \\
\operatorname{Tr}\left(M^{21}\right) & \operatorname{Tr}\left(M^{22}\right) & \operatorname{Tr}\left(M^{23}\right) \\
\operatorname{Tr}\left(M^{31}\right) & \operatorname{Tr}\left(M^{32}\right) & \operatorname{Tr}\left(M^{33}\right)
\end{array}\right) .
$$

Calculating the largest eigenvalue of this symmetric $3 \times 3$ matrix is inexpensive. Furthermore, the calculation only needs to be done once, before the 
start of the time stepping, because the material properties do not change in time. We then use $\kappa_{\max }$ as an approximation of $h^{2} \zeta / 4$, resulting in the time step restriction

$$
\frac{\Delta_{t}^{2}}{h^{2}} \kappa_{\max } \leq C_{c f l}^{2} .
$$

If the material model has heterogeneous properties, the procedure is repeated at each grid point of the mesh, and the largest value of $\kappa_{\max }$ is used in (59).

The Newmark scheme (54) is only second order accurate in time. The calculations shown in this paper use a predictor-corrector modification to obtain fourth order accuracy. It turns out that the fourth order scheme has a somewhat larger stability limit for the time step [11], but the procedure to estimate the largest eigenvalue $\zeta$ remains the same. Unless otherwise noted, we use $C_{c f l}=1.3$ in the numerical experiments in this paper.

\section{Super-grid boundary conditions}

We truncate unbounded or semi-bounded domains by using the supergrid approach $[27,12]$. In this technique, damping layers are added outside the domain of interest. The idea is to mimic a very large physical domain, where reflections from the boundary would need a very long time to return to the domain of interest. Similar to our treatment of curvilinear domains, a coordinate mapping is used in the layers. The elastic wave equation is discretized on a regular grid in parameter space, and the mapping corresponds to stretching the grid to cover a very large physical domain. In parameter space, the mapping acts by gradually slowing down and compressing the waves as they progress through the layer. A high order artificial dissipation operator is applied to damp out waves that become poorly resolved due to the coordinate mapping. The simple combination of a real-valued stretching function and artificial dissipation makes super-grid very straightforward to implement. This should be compared to the complexity of the PML method [17], where additional differential equations must be solved for auxiliary functions in the layers.

A very important property of the super-grid method is that, if the underlying scheme is stable on a curvilinear grid, it will also be stable with the artificial dissipation [12]. By using sufficiently smooth stretching functions and high order artificial damping terms, we demonstrated in [12] that, if the layers are sufficiently wide, artificial reflections can be made to converge to zero at the same rate as the interior scheme. In that paper we considered the isotropic elastic wave equation in heterogeneous materials. Here, we 
generalize the approach to the anisotropic case, where it is known that the PML technique can lead to stability problems [18].

For simplicity, we describe the super-grid technique for a Cauchy (wholespace) problem $\left(-\infty<x^{(k)}<\infty\right)$, with super-grid layers on all sides of the computational domain. This approach is straightforward to generalize to more general configurations by omitting the layers on some sides of the domain. The stretching functions are one-dimensional, i.e., $x^{(k)}=x^{(k)}\left(r^{(k)}\right)$ for $k=1,2,3$. Only the diagonal terms of the metric tensor $\xi_{i j}$ are non-zero and the curvilinear transformation is of the form

$$
\partial_{k}=\phi^{(k)}\left(r^{(k)}\right) \tilde{\partial}_{k}, \quad \phi^{(k)}=\frac{1}{\partial x^{(k)} / \partial r^{(k)}}, \quad k=1,2,3, \quad \xi_{i j}= \begin{cases}\phi^{(i)}, & i=j, \\ 0, & i \neq j .\end{cases}
$$

The Jacobian of the transformation satisfies $J^{-1}=\phi^{(1)} \phi^{(2)} \phi^{(3)}$.

An artificial dissipation term of order $2 p$ is added in the super-grid layers. On the semi-discrete level, the elastic wave equation with super-grid layers becomes

$$
\rho \frac{d^{2} \mathbf{u}}{d t^{2}}=\mathbf{L}_{h} \mathbf{u}+\mathbf{F}-\varepsilon(-1)^{p} \mathbf{Q}_{2 p}\left(\frac{d \mathbf{u}}{d t}\right), \quad \varepsilon=\gamma_{2 p} h^{2 p-1} \frac{c_{\max }}{C_{c f l}} .
$$

Here, $\gamma_{2 p}$ is a constant that depends on the order of the dissipation and $C_{c f l}$ is the CFL number that determines the time step for the fully discretized wave equation. For the isotropic elastic wave equation, we use $c_{\max }=$ $\sqrt{c_{p}^{2}+2 c_{s}^{2}}$, where $c_{p}$ and $c_{s}$ are the compressional and shear wave speeds, respectively. Section 4.3 discusses estimation of the wave speeds for the fully anisotropic equations. The dissipation term in (61) is of the form

$$
\mathbf{Q}_{2 p} \mathbf{v}=\left(\begin{array}{c}
\sum_{k=1}^{3} \phi^{(k)} Q_{2 p}^{(k)}\left(\sigma^{(k)} \rho\right) v^{(1)} \\
\sum_{k=1}^{3} \phi^{(k)} Q_{2 p}^{(k)}\left(\sigma^{(k)} \rho\right) v^{(2)} \\
\sum_{k=1}^{3} \phi^{(k)} Q_{2 p}^{(k)}\left(\sigma^{(k)} \rho\right) v^{(3)}
\end{array}\right)
$$

Each term in the sums of (62) acts along one of the coordinate directions, and $\sigma^{(k)}$ is a smoothly varying dissipation coefficient. The damping is scaled by density $(\rho)$ to make it balance the left hand side of (61). This allows $\gamma_{2 p}$ to be constant when $\rho$ varies in space.

We will use either fourth or sixth order artificial dissipation, corresponding to $p=2$ or $p=3$. When a fourth order $(p=2)$ artificial dissipation is used, each one-dimensional term is discretized according to

$$
Q_{4}^{(k)}\left(\sigma^{(k)} \rho\right) v:=D_{+}^{(k)} D_{-}^{(k)}\left(\sigma^{(k)} \rho D_{+}^{(k)} D_{-}^{(k)} v\right) .
$$


The sixth order $(p=3)$ artificial dissipation is discretized according to

$$
Q_{6}^{(k)}\left(\sigma^{(k)} \rho\right) v:=D_{+}^{(k)} D_{-}^{(k)} D_{+}^{(k)}\left(\bar{\sigma}^{(k)} \bar{\rho} D_{-}^{(k)} D_{+}^{(k)} D_{-}^{(k)} v\right)
$$

where cell-centered averages are used for the coefficients, e.g., $\bar{\sigma}_{j+1 / 2}=\left(\sigma_{j}+\right.$ $\left.\sigma_{j+1}\right) / 2$. Here $D_{ \pm}^{(k)}$ denote the standard first order forward and backward divided difference operators in direction $k$.

When (61) is discretized in time by the explicit predictor-corrector method, we want the stability restriction on the time step to be determined by the largest wave speed in the interior of the domain, and not by the amount of dissipation in the super-grid layers. For $C_{c f l}=1.3$, this turns out to be true for $\gamma_{4} \leq 0.02$ and $\gamma_{6} \leq 0.005$, respectively. If the damping coefficient exceeds these values, $C_{c f l}$ must be reduced to make the time-stepping stable, see $[12]$.

In [12] we used the energy method to prove stability of the discretized elastic wave equation with super-grid stretching and artificial dissipation. This can be done without the SBP boundary modifications at the damping layer boundaries. Instead, a sufficient number of ghost points are introduced such that the centered finite difference operators can be applied up to the outer boundary of the damping layer. Homogeneous Dirichlet conditions are imposed at all ghost points. This procedure leads to a SBP-like stability estimate, see [12] for details.

In practice the solution at the ghost points is set equal to zero before each time step. The material properties for the ghost points is usually extrapolated from the interior. Alternatively, they can be defined directly from the material model if it is defined on a sufficiently large domain.

The stretching function $\phi(r)$ and the damping functions $\sigma(r)$ are constructed from an auxiliary function $\psi_{w}(\xi)$ (the blue curve in Figure 2), which smoothly transitions from one to zero and then back to one,

$$
\psi_{w}(\xi)= \begin{cases}1, & \xi \leq(1-w) \ell \\ P((\ell-\xi) / w \ell), & (1-w) \ell<\xi<\ell \\ 0, & \ell \leq \xi \leq 1-\ell \\ P((\xi-(1-\ell)) / w \ell), & 1-\ell<\xi<1-(1-w) \ell \\ 1, & \xi \geq 1-(1-w) \ell\end{cases}
$$

where $0<w \leq 1$ is the relative width of the transition. We use the polynomial function $P(\eta)=\eta^{6}\left(462-1980 \eta+3465 \eta^{2}-3080 \eta^{3}+1386 \eta^{4}-252 \eta^{5}\right)$, which satisfies $P(0)=0, P(1)=1$, and makes $\psi_{w}(\xi)$ five times continuously 


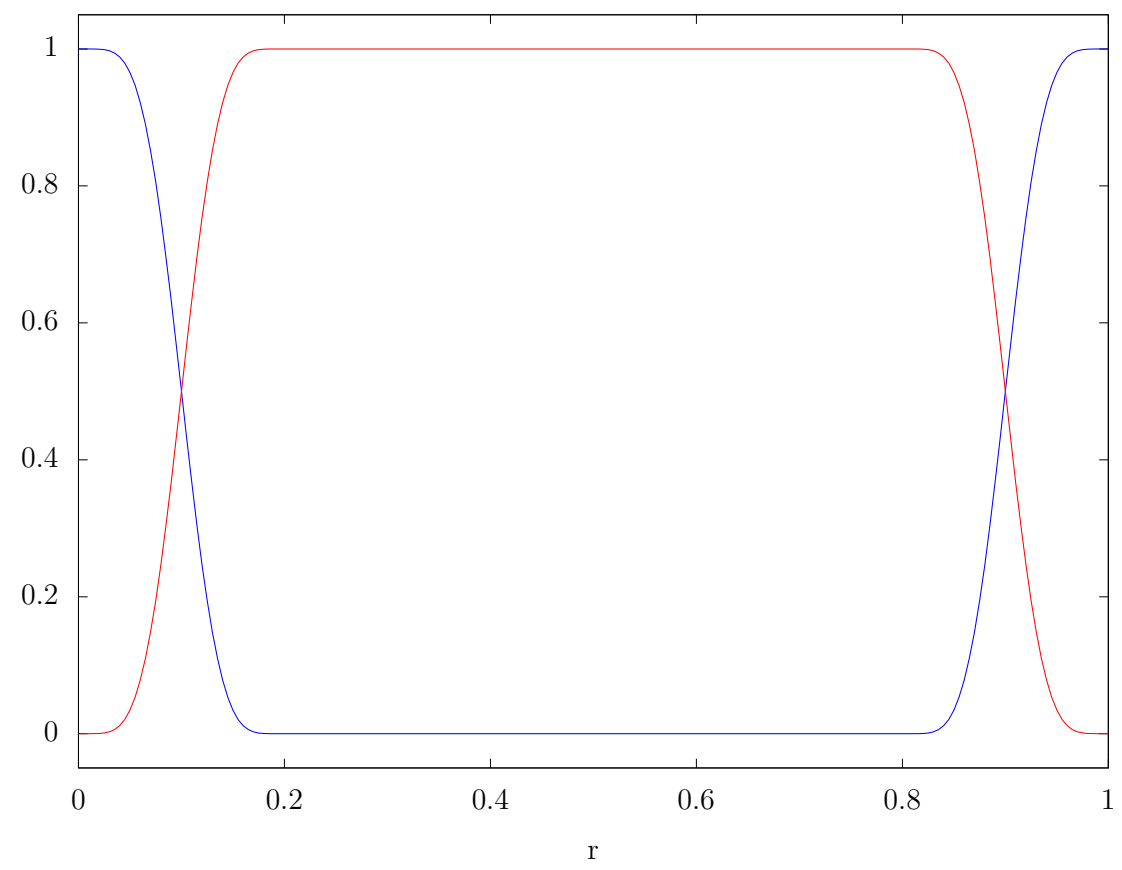

Figure 2: The stretching function $\phi(r)$ (red) and the auxiliary function $\psi(r)$ (blue), which controls the strength of the damping. In this case, the width of each super-grid layer is $\ell=0.2$.

differentiable. The one-dimensional stretching and damping functions are defined by

$$
\phi(r)=\left(1-\left(1-\varepsilon_{L}\right) \psi_{1}(r)\right), \quad \sigma(r)=\frac{\psi_{w}(r)}{\phi(r)}, \quad 0<\varepsilon_{L} \ll 1 .
$$

This means that $\phi(r)=1$, and $\sigma(r)=0$ for $\ell \leq r \leq 1-\ell$. Note that $\varepsilon_{L}$ is given a small positive value to prevent the coordinate stretching from becoming singular. It is not related to the damping coefficient $\varepsilon$ in (61). Throughout the numerical experiments in this paper, we use $\varepsilon_{L}=10^{-4}$. The relative transition width for $\phi$ is fixed to one, while we use $w=0.5$ or $w=1.0$ for $\sigma$. Examples of the functions $\psi$ and $\phi$ are plotted in Figure 2.

\subsection{Three-dimensional considerations}

Along the sides of a three-dimensional domain, where only one supergrid damping layer is active, we use a one-dimensional damping function, as 
described above. For example,

$$
\sigma^{(1)}\left(r^{(1)}, r^{(2)}, r^{(3)}\right)=\sigma\left(r^{(1)}\right), \quad 0 \leq r^{(1)} \leq \ell, \quad \ell \leq\left(r^{(2)}, r^{(3)}\right) \leq 1-\ell .
$$

If the one-dimensional damping function is used where several super-grid layers meet (at edges or corners of the computational domain), it is necessary to reduce the damping coefficient $\left(\gamma_{2 p}\right)$ to avoid making the explicit timestepping scheme unstable, see [12]. However, this reduces the strength of the damping where only one super-grid layer is active, which leads to larger artificial reflections.

A simple remedy is provided by introducing the linear taper function $\tau$,

$$
\tau(r)= \begin{cases}\alpha, & r<0 \\ \alpha+(1-\alpha) r / \ell, & 0 \leq r \leq \ell \\ 1, & r>\ell\end{cases}
$$

For example, along the edge $0 \leq r^{(1)} \leq \ell, 0 \leq r^{(2)} \leq \ell$, we define the two-dimensional damping functions

$$
\left\{\begin{array}{l}
\sigma^{(1)}\left(r^{(1)}, r^{(2)}, r^{(3)}\right)=\sigma\left(r^{(1)}\right) \tau\left(r^{(2)}\right), \\
\sigma^{(2)}\left(r^{(1)}, r^{(2)}, r^{(3)}\right)=\tau\left(r^{(1)}\right) \sigma\left(r^{(2)}\right),
\end{array} \quad \ell \leq r^{(3)} \leq 1-\ell,\right.
$$

where $\sigma(r)$ is the one-dimensional damping function (66). Using this construction, the strength of the damping is determined by

$$
I_{2}\left(r^{(1)}, r^{(2)}\right):=\left(\sigma^{(1)}+\sigma^{(2)}\right) \phi=\tau\left(r^{(2)}\right) \psi\left(r^{(1)}\right)+\tau\left(r^{(1)}\right) \psi\left(r^{(2)}\right),
$$

where $\psi(r)$ is the auxiliary function (65). When $\alpha=1 / 3$, this construction satisfies $\max I_{2}=1$. Away from the edge, the strength of the damping is the same as in the one-dimensional case because $\psi(r)=0$ and $\tau(r)=1$ for $r \geq \ell$. Therefore, $I_{2}\left(r^{(1)}, r^{(2)}\right)=\psi\left(r^{(2)}\right)$ for $r^{(1)} \geq \ell$ and $I\left(r^{(1)}, r^{(2)}\right)=\psi\left(r^{(1)}\right)$ for $r^{(2)} \geq \ell$. At the edge, $\tau(0)=1 / 3$ and $\psi(0)=1$, giving $I_{2}(0,0)=2 / 3$. The function $I_{2}\left(r^{(1)}, r^{(2)}\right)$ has a local maxima along the diagonal $r^{(1)}=$ $r^{(2)} \approx 0.31 \ell$, where $I_{2} \approx 0.983$. The tapering approach is straightforward to generalize to the other edges of the computational domain.

Near a corner where three super-grid layers meet, the strength of the damping equals $I_{3}:=\left(\sigma^{(1)}+\sigma^{(2)}+\sigma^{(3)}\right) \phi$. For $r^{(k)} \leq \ell$, we generalize the tapering approach by defining

$$
\left\{\begin{array}{l}
\sigma^{(1)}\left(r^{(1)}, r^{(2)}, r^{(3)}\right)=\sigma\left(r^{(1)}\right) \tau\left(r^{(2)}\right) \tau\left(r^{(3)}\right), \\
\sigma^{(2)}\left(r^{(1)}, r^{(2)}, r^{(3)}\right)=\tau\left(r^{(1)}\right) \sigma\left(r^{(2)}\right) \tau\left(r^{(3)}\right), \\
\sigma^{(3)}\left(r^{(1)}, r^{(2)}, r^{(3)}\right)=\tau\left(r^{(1)}\right) \tau\left(r^{(2)}\right) \sigma\left(r^{(3)}\right) .
\end{array}\right.
$$


This construction also satisfies $\max I_{3}=1$. The strength of the damping has a local maxima along the space-diagonal $r^{(1)}=r^{(2)}=r^{(3)} \approx 0.37 \ell$ where $I_{3} \approx 0.823$. Also note that the two-dimensional strength is recovered along edges of the three-dimensional domain (where two super-grid layers meet), e.g. $I_{3}\left(r^{(1)}, r^{(2)}, r^{(3)}\right)=I_{2}\left(r^{(1)}, r^{(2)}\right)$ for $r^{(3)} \geq \ell$.

The tapering approach is of significant practical importance in threedimensional calculations, where up to three super-grid layers can meet at corners. This is because the tapering keeps the maximum strength of the super-grid damping approximately equal along sides, edges, and corners of the computational domain. With the tapering approach, if the damping coefficient $\gamma_{2 p}$ makes the time stepping stable with super-grid damping in only one direction, the same value will also work when three super-grid layers meet at a corner. Without the tapering approach, the time stepping would become unstable unless the damping coefficient is reduced to approximately $\gamma_{2 p} / 3$, leading to less efficient damping. To compensate, the layers would need to be much thicker. Since the super-grid layers are added outside the domain of interest, this would significantly increase the total number of grid points in a three-dimensional case, thus making the calculation much more expensive.

\section{Numerical experiments}

All simulations reported here were performed with the open source code SW4, version 1.1 [28]. Our theory and numerical method are applicable to any heterogeneous anisotropic material as long as the stiffness matrix is positive definite and the density is positive. In Section 6.1, we use the method of manufactured solutions (see, e.g. [29]) to check the order of accuracy in the numerical solution when both the material properties and the solution are smooth. In Section 6.2, we evaluate the energy conservation property in a heterogeneous material with piecewise constant properties. We exemplify wave propagation properties in a homogeneous anisotropic material in Section 6.3, followed by evaluating the reflection properties of the super-grid layers in Section 6.4. Our final numerical example illustrates anisotropic wave propagation in a half-space, subject to a free surface boundary condition on one side of the domain and truncated by super-grid layers on all other sides, see Section 6.5.

\subsection{Method of manufactured solutions}

We take the computational domain to be $0 \leq x^{(k)} \leq 6, k=1,2,3$, with a free surface boundary conditions on the $x^{(3)}=0$ boundary and Dirichlet 


\begin{tabular}{|c|c|c|c|c|c|}
\hline$N_{x}$ & $h$ & $\left\|\mathbf{u}(\cdot, t)-\mathbf{u}_{e}(\cdot, t)\right\|_{\infty}$ & $\left\|\mathbf{u}(\cdot, t)-\mathbf{u}_{e}(\cdot, t)\right\|_{2}$ & $p_{\infty}$ & $p_{2}$ \\
\hline 31 & $2.0 \cdot 10^{-1}$ & $1.57 \cdot 10^{-3}$ & $3.38 \cdot 10^{-3}$ & - & - \\
\hline 61 & $1.0 \cdot 10^{-1}$ & $8.15 \cdot 10^{-5}$ & $1.67 \cdot 10^{-4}$ & 4.26 & 4.33 \\
\hline 121 & $5.0 \cdot 10^{-2}$ & $4.27 \cdot 10^{-6}$ & $9.19 \cdot 10^{-6}$ & 4.25 & 4.18 \\
\hline 241 & $2.5 \cdot 10^{-2}$ & $2.73 \cdot 10^{-7}$ & $5.35 \cdot 10^{-7}$ & 3.96 & 4.10 \\
\hline 481 & $1.25 \cdot 10^{-2}$ & $1.65 \cdot 10^{-8}$ & $3.23 \cdot 10^{-8}$ & 4.04 & 4.04 \\
\hline
\end{tabular}

Table 1: Errors in the numerical solution at time $t=1.0$, on a uniform grid with $h=$ $6 /\left(N_{x}-1\right)$, when the exact solution is (67). Here, $p_{\infty}$ and $p_{2}$ are the convergence exponents in the maximum norm and 2-norm, respectively.

conditions on all other boundaries. Let the density vary according to

$$
\rho=A_{\rho}\left(2+\sin \left(\omega_{m} x^{(1)}+\theta_{\rho}\right) \cos \left(\omega_{m} x^{(2)}+\theta_{\rho}\right) \sin \left(\omega_{m} x^{(3)}+\theta_{\rho}\right)\right),
$$

where $A_{\rho}=1.5, \omega_{m}=0.8$, and $\theta_{\rho}=0.3$. The elements of the symmetric stiffness matrix satisfy

$$
c_{i j}=A_{i j}+\sin \left(\omega_{m} x^{(1)}+\theta_{i j}^{(1)}\right) \cos \left(\omega_{m} x^{(2)}+\theta_{i j}^{(2)}\right) \sin \left(\omega_{m} x^{(3)}+\theta_{i j}^{(3)}\right), \quad j \geq i,
$$

where $A_{i j}=2$ for $i \neq j$ and $A_{j j}=10$. The values of the phase angles $\theta_{i j}^{(k)}$ are different for each of the 21 elements of the stiffness matrix (not shown to conserve space). The internal forcing, boundary forcing and initial conditions are chosen such that the exact (manufactured) solution becomes

$$
\begin{aligned}
& u^{(1)}=\sin \left(\omega\left(x^{(1)}-c_{v} t\right)\right) \sin \left(\omega x^{(2)}+\phi\right) \sin \left(\omega x^{(3)}+\phi\right), \\
& \left.u^{(2)}=\sin \left(\omega x^{(1)}+\phi\right)\right) \sin \left(\omega\left(x^{(2)}-c_{v} t\right)\right) \sin \left(\omega x^{(3)}+\phi\right), \\
& \left.u^{(3)}=\sin \left(\omega x^{(1)}+\phi\right)\right) \sin \left(\omega x^{(2)}+\phi\right) \sin \left(\omega\left(x^{(3)}-c_{v} t\right)\right) .
\end{aligned}
$$

Here, $\omega=1.2, c_{v}=1.3$, and $\phi=0.8$. Table 1 gives the errors in the numerical solution, evaluated in maximum norm and $L^{2}$ norm at different grid resolutions at time $t=1.0$. The maximum norm is computed over all grid points in the domain and over the three components of the solution. Similarly, the discrete $L^{2}$-norm is computed both over the domain and over the three solution components. In both norms, the numerical solution convergences towards the exact solution as $\mathcal{O}\left(h^{4}\right)$. 


\subsection{Energy conservation test}

To verify the theoretically predicted energy conservation property of our scheme, we perform a computation without source terms, but with uniformly distributed random noise as initial data. For this calculation, the computational domain is $0 \leq x^{(1)} \leq 3 \cdot 10^{4}, 0 \leq x^{(2)} \leq 3 \cdot 10^{4}, 0 \leq x^{(3)} \leq 1.7 \cdot 10^{4}$. Energy conservation is ensured by enforcing periodic boundary conditions in the $x^{(1)}$ - and $x^{(2)}$-directions, a free surface boundary condition along $x^{(3)}=0$, and a homogeneous Dirichlet condition along $x^{(3)}=1.7 \cdot 10^{4}$. The grid spacing is $h=200$, which gives $151 \times 151 \times 86$ grid points. We use a vertically layered material model, with Indium Arsenide (InAs) in the subdomain $x^{(3)} \leq 2 \cdot 10^{3}$ and quartz in $x^{(3)}>2 \cdot 10^{3}$. InAs is a crystal with cubic symmetry. When the coordinate axes are oriented along the bonds of the cubic crystal, its density and stiffness matrix are given by (see [30])

$$
\rho=5.67 \cdot 10^{3}, C=\left(\begin{array}{cccccc}
83.29 & 45.26 & 45.26 & 0 & 0 & 0 \\
45.26 & 83.29 & 45.26 & 0 & 0 & 0 \\
45.26 & 45.26 & 83.29 & 0 & 0 & 0 \\
0 & 0 & 0 & 39.59 & 0 & 0 \\
0 & 0 & 0 & 0 & 39.59 & 0 \\
0 & 0 & 0 & 0 & 0 & 39.59
\end{array}\right) \cdot 10^{9},
$$

using SI-units. Quartz is a trigonal material that is modeled by the density and stiffness matrix,

$$
\rho=2.62 \cdot 10^{3}, C=\left(\begin{array}{cccccc}
86.74 & 6.99 & 6.99 & -17.91 & 0 & 0 \\
6.99 & 86.74 & 6.99 & 17.91 & 0 & 0 \\
6.99 & 6.99 & 107.2 & 0 & 0 & 0 \\
-17.91 & 17.91 & 0 & 57.94 & 0 & 0 \\
0 & 0 & 0 & 0 & 57.94 & -17.91 \\
0 & 0 & 0 & 0 & -17.91 & 39.875
\end{array}\right) \cdot 10^{9} \text {, }
$$

also using SI-units.

For the semi-discrete approximation, the energy is given by $\left(\rho u_{t}, u_{t}\right)_{h}+$ $S_{h}(u, u)$, see (53). By using the same approach as for the isotropic elastic wave equation, see [11], the expression for the fully discrete energy becomes

$$
E_{h}^{n+1 / 2}=\left\|\sqrt{\rho} \frac{\mathbf{u}^{n+1}-\mathbf{u}^{n}}{\Delta_{t}}\right\|_{h}^{2}+S_{h}\left(\mathbf{u}^{n+1}, \mathbf{u}^{n}\right)-\frac{\Delta_{t}^{2}}{12}\left(\mathbf{L}_{h} \mathbf{u}^{n+1}, \mathbf{L}_{h} \mathbf{u}^{n}\right)_{h} .
$$




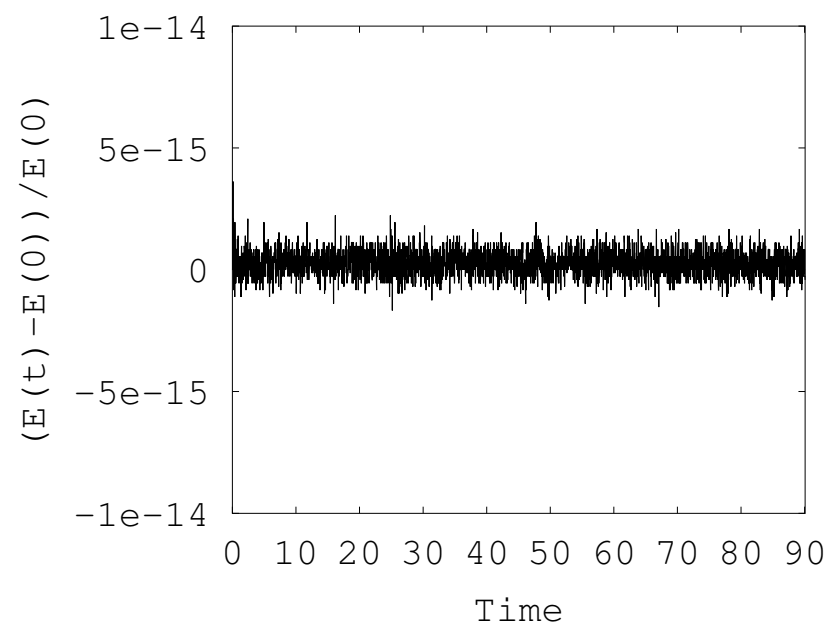

Figure 3: The relative change in discrete energy as function of time, with random initial data and a heterogeneous layered anisotropic material model. Here, time $t=90$ corresponds to 3,200 time steps.

In Figure 3 we plot the relative change in discrete energy, $\left(E^{n+1 / 2}-E^{1 / 2}\right) / E^{1 / 2}$ as function of time, for $t \in[0,90]$. This corresponds to 3,200 time steps. Our calculation confirms that the discrete energy remains constant modulus very small fluctuations, which are on the order of the round-off level in double precision arithmetic.

\subsection{Wave propagation in anisotropic materials}

The fundamental properties of wave propagation in a homogeneous anisotropic material can be quantified by its phase and group velocities, as well as its slowness surfaces, see e.g. [20] for details. A plane wave propagating in unit direction $\mathbf{n}=\left(n_{1}, n_{2}, n_{3}\right)^{T},|\mathbf{n}|=1$, can be described by the equivalent formulas

$$
\mathbf{u}(\mathbf{x}, t)=\mathbf{r} e^{i(\mathbf{k} \cdot \mathbf{x}-\omega t)}=\mathbf{r} e^{i \xi(\mathbf{n} \cdot \mathbf{x}-c t)}=\mathbf{r} e^{i \omega(\mathbf{s} \cdot \mathbf{x}-t)} .
$$

Here, $\mathbf{r}$ is the polarization vector, $\mathbf{k}=\left(k_{1}, k_{2}, k_{3}\right)^{T}=\xi \mathbf{n}$ is the wave vector, $\omega$ the angular frequency, $\xi$ the (angular) wave number, and $\mathbf{s}=\mathbf{k} / \omega$ is the slowness vector. Assuming homogeneous material properties, inserting the plane wave ansatz into the elastic wave equation (1) with $\mathbf{F}=\mathbf{0}$ and using (5), leads to the dispersion relation (57), i.e., the Christoffel equation. The 


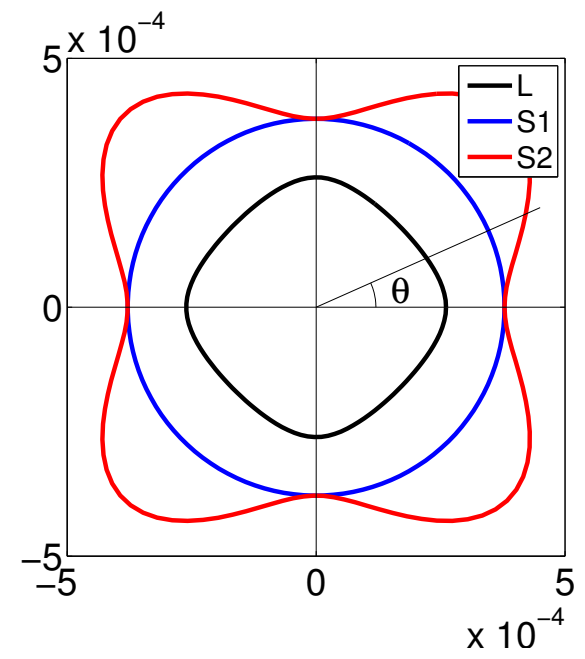

Figure 4: Slowness curves for InAs for wave propagation in the $\left(x^{(1)}, x^{(2)}\right)$ plane. Units are in $[s / m]$.

phase velocity,

$$
c=\frac{\omega}{\xi}=\frac{1}{|\mathbf{s}|},
$$

equals the (positive) square root of an eigenvalue $c^{2}$ of the Christoffel equation. Note that the matrix in (57) is symmetric and positive definite, so $c^{2}$ is real and positive.

A slowness surface is defined by $\mathbf{s}=\mathbf{n} / c$. For each direction $\mathbf{n}$, the Christoffel equation has three eigenvalues, corresponding to three slowness surfaces. A slowness curve is a cross-section of a slowness surface. In Figure 4 we show the slowness curves for InAs in the $\left(x^{(1)}, x^{(2)}\right)$ plane. The curves are plotted in polar coordinates $(r, \theta)$. For each angle $\theta$, we solve the Christoffel equation in the direction $\left(n^{(1)}, n^{(2)}, n^{(3)}\right)=(\cos \theta, \sin \theta, 0)$, resulting in three eigenvalues $c_{k}^{2}(\theta), k=1,2,3$. The corresponding radii in polar coordinates are $r_{k}(\theta)=1 / c_{k}(\theta)$. The innermost curve (black color, labeled "L") corresponds to the quasi-longitudinal wave. It has the largest phase velocity, which only varies mildly with the direction of wave propagation. The second fastest wave is shown in blue color and labeled "S1". This is a quasi-transverse wave with the same phase velocity in all directions, corresponding to a circular slowness curve. The other quasi-transverse wave (red color, labeled "S2") has the slowest phase velocity, with the minima $c_{\text {min }}=1.831 \cdot 10^{3}$ at $\theta=\pi / 4,3 \pi / 4,5 \pi / 4$, and $7 \pi / 4$. 
To illustrate some properties of anisotropic wave propagation, we numerically solve the elastic wave equation in a homogeneous InAs crystal, truncated by super-grid layers on all sides of the domain. We take the domain of interest to be $\left(x^{(1)}, x^{(2)}, x^{(3)}\right) \in\left[1.6 \cdot 10^{3}, 10.4 \cdot 10^{3}\right]^{3}$. In the unit cube of parameter space, the super-grid layers have thickness $\ell=1.6 / 12 \approx 0.133$. In the figures below, cross-sections of the solution are plotted as function of scaled parameter coordinates, to equal $\left(x^{(1)}, x^{(2)}, x^{(3)}\right)$ within the domain of interest. In this scaled parameter space, the super-grid layers have thickness $1.6 \cdot 10^{3}$.

The solution is driven by an isotropic point moment tensor source,

$$
\mathbf{f}(\mathbf{x}, t)=g(t) M_{0}\left(\begin{array}{lll}
1 & 0 & 0 \\
0 & 1 & 0 \\
0 & 0 & 1
\end{array}\right) \nabla \delta\left(\mathbf{x}-\mathbf{x}_{s}\right), \quad M_{0}=10^{17}
$$

located at $\mathbf{x}_{s}=(6,6,6) \cdot 10^{3}$. Here, $\nabla \delta$ is the gradient of the Dirac distribution, which is discretized in space by using the technique described in [31]. The source time function is the Gaussian,

$$
g(t)=\frac{1}{\sqrt{2 \pi \sigma}} e^{\left(t-t_{0}\right)^{2} / 2 \sigma^{2}}, \quad \sigma=\frac{1}{16}, \quad t_{0}=0.375 .
$$

We estimate the dominant frequency in the Gaussian by $f_{0}=1 /(2 \pi \sigma) \approx$ 2.55 and the highest significant frequency by $f_{\max } \approx 2.5 f_{0} \approx 6.37$.

For wave propagation problems, the resolution requirements for a finite difference discretization can be quantified in terms of the number of grid points per shortest wave length, $P=L_{\min } / h$, see [32]. Based on the largest significant frequency of the Gaussian $\left(f_{\max } \approx 6.37\right)$, and the slowest shear velocity $\left(c_{\min }=1831\right)$, we estimate the shortest shear wave length to be $L_{\min }=c_{\min } / f_{\max } \approx 1831 / 6.37 \approx 287.6$. In the numerical experiments below, we use grid sizes $h=40,20$, and 10 corresponding to $P=7.19$, 14.38 , and 28.76 grid points per shortest significant wave length.

In Figure 5 we show the magnitude of the displacement in the $x^{(3)}=$ $6 \cdot 10^{3}$ plane at time $t=1.5$. The outermost wave front corresponds to the quasi-compressional wave. Corresponding to the shape of its slowness curve (labeled "L" in Figure 4), it propagates slightly faster along the diagonal than along the coordinate axes. The waves closer to the center of the figure are of quasi-shear type, which are generated by the moment tensor source (69), even though it is isotropic. The complex shape of the wave fronts is a result of the directional variation in phase velocity. Because the 


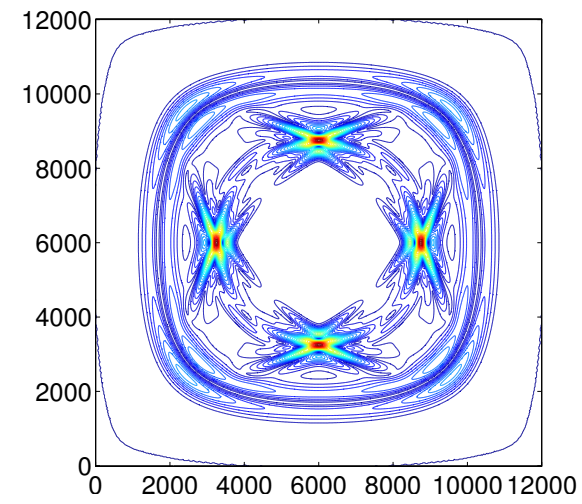

Figure 5: Magnitude of the displacement at time $t=1.5$ in the plane $x^{(3)}=6000$. The contour levels are spaced between 0.0375 (dark blue) and 1.2 (red) with step size 0.0375.

motion is generated by a point source, all wave fronts are initially circular, but the quasi-shear waves quickly develop a more complicated structure. In particular, note the swallow tail-shapes of the slowest quasi-shear wave. They are due to the inflection points in the "S2" slowness curve in Figure 4.

In Figure 6 we show the Cartesian components of the solution for $t \in$ $[0,6]$, at the location $\mathbf{x}_{r}=(2.0,3.6,5.2)^{T} \cdot 10^{3}$, which is near a super-grid layer. The difference between solutions computed with grid sizes $h=20$ and $h=10$ is shown on the right side of the same figure. Note that the difference is significantly smaller than the solution itself, indicating that it is well-resolved on the grid. Also note that no artificial reflections are visible in the left plot of Figure 6.

\subsection{Absorption properties of the super-grid layers}

To choose the width and other parameters of the super-grid layers we want to numerically evaluate the amount of artificial reflections due to the far-field truncation. As a challenging test case, we continue studying wave propagation in a homogeneous InAs crystal. According to the theory by Bécache et al. [18], this material violates the geometric stability condition [19] for a perfectly matched layer (PML), because the slowness curve of the quasi-shear wave "S2" has several segments where one component of the slowness and the group velocity vectors have opposite signs. As a result, a numerical approximation using a perfectly matched layer (PML) can become unstable.

In [12] we evaluated reflection errors from the super-grid layers in an 

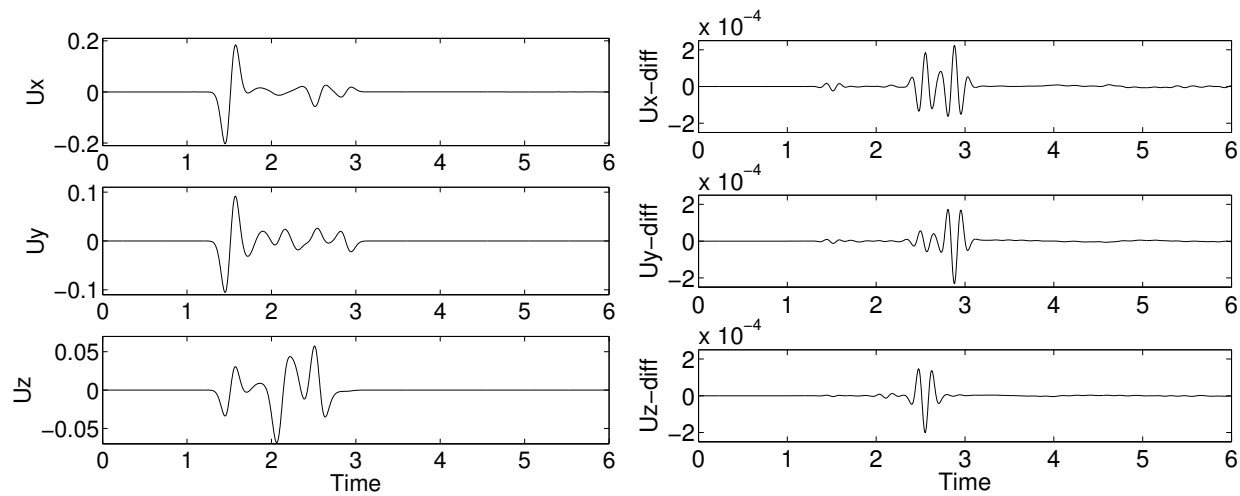

Figure 6: Solution of the Cauchy (whole-space) problem at $\left(x^{(1)}, x^{(2)}, x^{(3)}\right)=(2.0,3.6,5.2)$. $10^{3}$ as function of time, computed with grid size $h=20$ (left). Difference between the numerical solutions computed with grid size $h=20$ and $h=10$ (right).

isotropic elastic material by solving Lamb's problem in a half-space, which has an analytical solution that allows the error to be evaluated explicitly. Because the anisotropic elastic wave equation is very difficult to solve analytically, we instead exploit the absence of evanescent modes in the solution of a Cauchy (whole-space) problem with homogeneous material properties. This means that the analytical solution due to a point source is identically zero after all waves have propagated past a fixed location in space. After that time, the numerical solution therefore equals the reflection error.

Because it is impractical to store the numerical solution at all points in space and time, we limit our investigation to study the time-dependent solution at fixed locations in the outer parts of the domain of interest. We use the same setup as in Section 6.3 with the source specified by (69)-(70) and located in the same place. For each grid size, we record the solution (as function of time) at nine locations, on a uniform $3 \times 3$ grid,

$$
x_{r}^{(1)}=2 \cdot 10^{3}, \quad x_{r}^{(2)}=(2.0,3.6,5.2) \cdot 10^{3}, \quad x_{r}^{(3)}=(2.0,3.6,5.2) \cdot 10^{3} .
$$

We proceed by studying the norm of the displacement,

$$
|\mathbf{u}|\left(\mathbf{x}_{r}, t\right)=\sqrt{\sum_{k=1}^{3}\left(u^{(k)}\right)^{2}\left(\mathbf{x}_{r}, t\right)},
$$

at the nine locations (71) for $t \geq t_{1}$. 

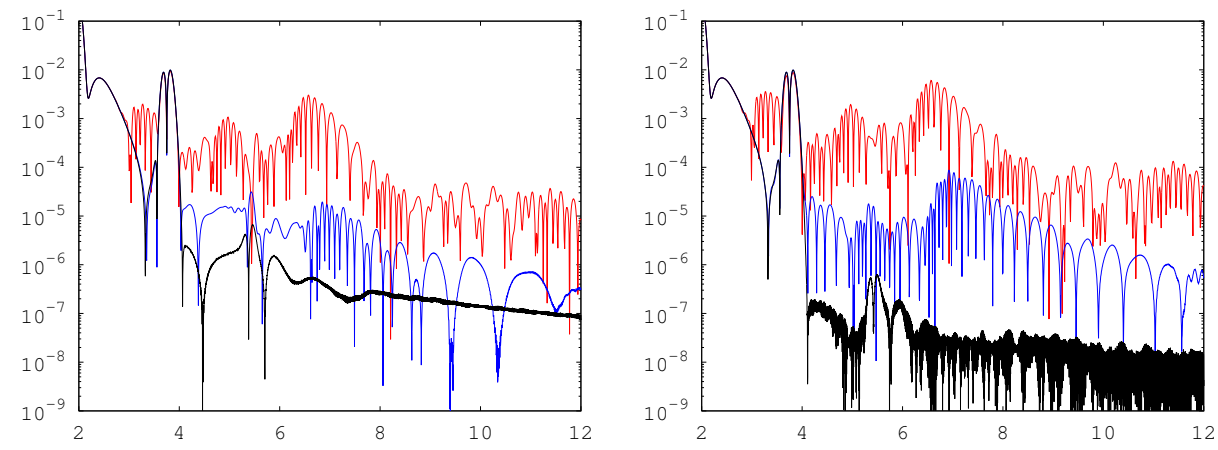

Figure 7: Norm of the numerical solution at $\mathbf{x}_{r}=(2.0,2.0,2.0) \cdot 10^{3}$ as function of time, for grid sizes $h=40$ (red), $h=20$ (blue), and $h=10$ (black). The artificial dissipation is of order four and six in the left and right sub-figures, respectively.

Of the nine locations in $(71), \mathbf{x}_{r}=(2.0,2.0,2.0) \cdot 10^{3}$ is the furthest from the source, at a distance $d \approx 6.928 \cdot 10^{3}$. The slowest phase velocity is $c_{\min }=$ $1.831 \cdot 10^{3}$, from which we estimate the propagation time from the source to $\mathbf{x}_{r}$ to be $t_{p} \leq 6.928 / 1.831 \approx 3.784$. The Gaussian source time function (70) satisfies $g(t) \leq 10^{-7}$ for $t \geq 2 t_{0}=0.75$ (note that the Gaussian time function decays exponentially fast for large times, but is never identically zero). By combining these estimates, we conclude that the analytical solution should reach round-off levels after $t \geq t_{1} \approx 3.784+0.75=4.534$. To test this estimate, we plot the norm of the displacement at $\mathbf{x}_{r}=(2.0,2.0,2.0) \cdot 10^{3}$ in Figure 7 . On the finest grid, the solution decays from $|\mathbf{u}|\left(\mathbf{x}_{r}, t\right) \approx 10^{-2}$ for $t \approx 3.9$ to $|\mathbf{u}|\left(\mathbf{x}_{r}, t\right)<10^{-5}$ for $t \geq 4.1$. This rapid decay indicates that the analytical solution can be taken to be zero for $t \geq t_{2}$, where $t_{2} \approx 4.1$. The fact that $t_{1}>t_{2}$ indicates that we underestimated the value of the slowest phase velocity in the direction between the source and $\mathbf{x}_{r}$.

In Figure 7 we also compare the difference between fourth and sixth order artificial dissipation in the super-grid layer, when its width is kept constant. By studying the norm of the numerical solution for $t \geq 4$.1 we conclude that the sixth order dissipation gives smaller reflection errors on the finest grid (black curves). However, the fourth order dissipation gives comparable, or slightly smaller, reflection errors on the two coarser grids (blue and red curves). The numerical solutions at the remaining locations (given by (71)) show the same qualitative behavior. Based on these limited numerical experiments, we surmise that the benefits of using a sixth order artificial dissipation are very limited. Furthermore, the code for the sixth 


\begin{tabular}{|c|c||c|}
\hline$N_{s g}$ & $w$ & $\max _{r} e\left(\mathbf{x}_{r}\right)$ \\
\hline 20 & 1.0 & $6.545 \mathrm{e}-2$ \\
\hline 30 & 1.0 & $2.314 \mathrm{e}-2$ \\
\hline 40 & 1.0 & $1.117 \mathrm{e}-2$ \\
\hline 50 & 1.0 & $4.367 \mathrm{e}-3$ \\
\hline
\end{tabular}

\begin{tabular}{|c|c||c|}
\hline$N_{s g}$ & $w$ & $\max _{r} e\left(\mathbf{x}_{r}\right)$ \\
\hline 20 & 0.5 & $4.762 \mathrm{e}-2$ \\
\hline 30 & 0.5 & $1.539 \mathrm{e}-2$ \\
\hline 40 & 0.5 & $6.143 \mathrm{e}-3$ \\
\hline 50 & 0.5 & $2.235 \mathrm{e}-3$ \\
\hline
\end{tabular}

Table 2: Relative reflection errors from the super-grid layers for a Cauchy (whole-space) problem with the relative transition width $w=1$ (left) and $w=0.5$ (right) in the damping coefficient profile (66). The largest relative reflection error, defined by (73), of the nine stations is reported here. The width of the super-grid layers are $h N_{s g}$, where $h=40$.

order dissipation is slightly slower than its fourth order counterpart because it requires a wider computational stencil and more data to be communicated after each time step. Hence, the fourth order artificial dissipation appears to be preferable for most practical simulations, which seldom use more than 15 grid points per shortest wave length. The remainder of our numerical experiments use the fourth order artificial dissipation.

In our next numerical experiment we keep the same general setup as before, but vary the number of grid points in the super-grid layers. The size of the domain of interest is kept the same and we also use the same locations of the receivers, relative to the point source. Here we quantify the reflection errors by evaluating how large the norm of the numerical solution is in the time interval $4.5 \leq t \leq 8.0$, relative to its maximum value for $0 \leq t \leq 8.0$. Here the norm of the solution is computed according to (72), and the relative reflection error at each station is defined by

$$
e\left(\mathbf{x}_{r}\right)=\frac{\max _{4.5 \leq t \leq 8}|\mathbf{u}|\left(\mathbf{x}_{r}, t\right)}{\max _{0 \leq t \leq 8}|\mathbf{u}|\left(\mathbf{x}_{r}, t\right)}
$$

On the left side of Table 2 we report the largest value of $e\left(\mathbf{x}_{r}\right)$ from the nine stations when the grid size is $h=40$. Our results indicate that the reflection errors decrease substantially by making the layers wider. To further reduce the reflections, we increase the amount of damping in the middle of each layer by changing the relative transition width $(w)$ in the damping coefficient $\sigma(r)$, defined by (66). Note that the stretching function, $\phi(r)$, is kept the same as before. After some numerical experimentation, we found that the time stepping algorithm remains stable for $\gamma_{4}=0.02$ and $C_{c f l}=1.3$, if the transition width satisfies $0.4 \leq w \leq 1$. In a second set of numerical experiments, we repeat the above calculations but set $w=0.5$, 


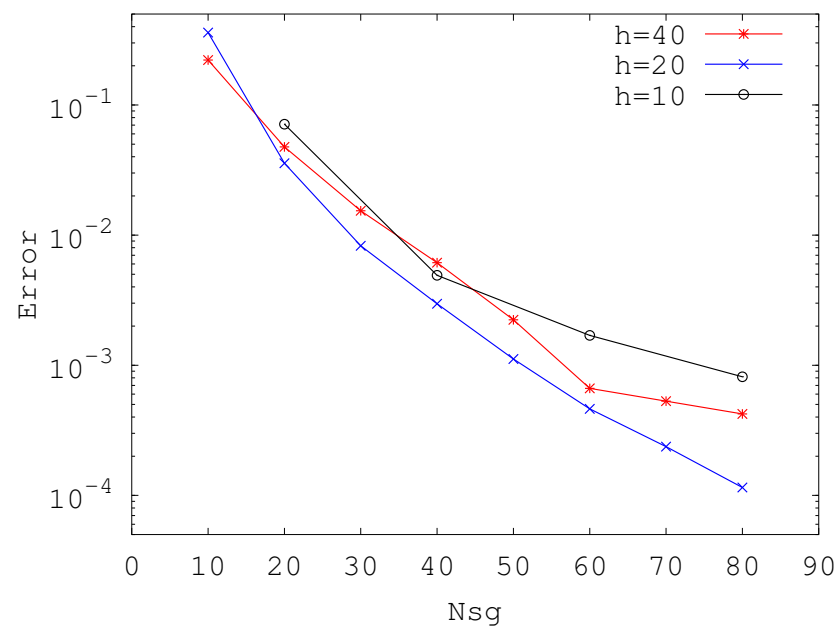

Figure 8: Relative reflection errors as function of the number of grid points $\left(N_{s g}\right)$ in each super-grid layer.

see the right side of Table 2. We conclude that the reflection errors are reduced by a factor of almost two compared to $w=1$. In the remainder of the experiments of this section, we use the relative transition width $w=0.5$.

To further investigate the reflection errors from the super-grid layers, we repeat the above experiment for different grid sizes. In Figure 8 we show the relative reflection error as function of the number of grid points in the super-grid layers. This means that the width of the layer decreases as the grid is refined. Here no improvement of the reflection error is observed as the grid is refined. In fact, the errors are consistently smaller for $h=20$ compared to $h=10$.

Another, more expensive, alternative is to keep the width of the supergrid layer fixed as the grid is refined. This means that the number of points in the layers is doubled every time the grid size if halved. In Figure 9 we show the reflection error as function of the width $N_{s g} h$, for the three grid sizes $h=40, h=20$, and $h=10$. Here we observe a drastic decrease in reflection error between $h=40$ and $h=20$, while there is about a factor of 8 reduction in the error between $h=20$ and $h=10$. We speculate that the factor of 8 is due to the fourth order artificial dissipation, which is of strength $\mathcal{O}\left(h^{3}\right)$. Further numerical experiments would be needed to see if the reflection errors can be made to decrease as $\mathcal{O}\left(h^{4}\right)$ with a sixth order artificial dissipation in the super-grid layers, thus making the reflection 


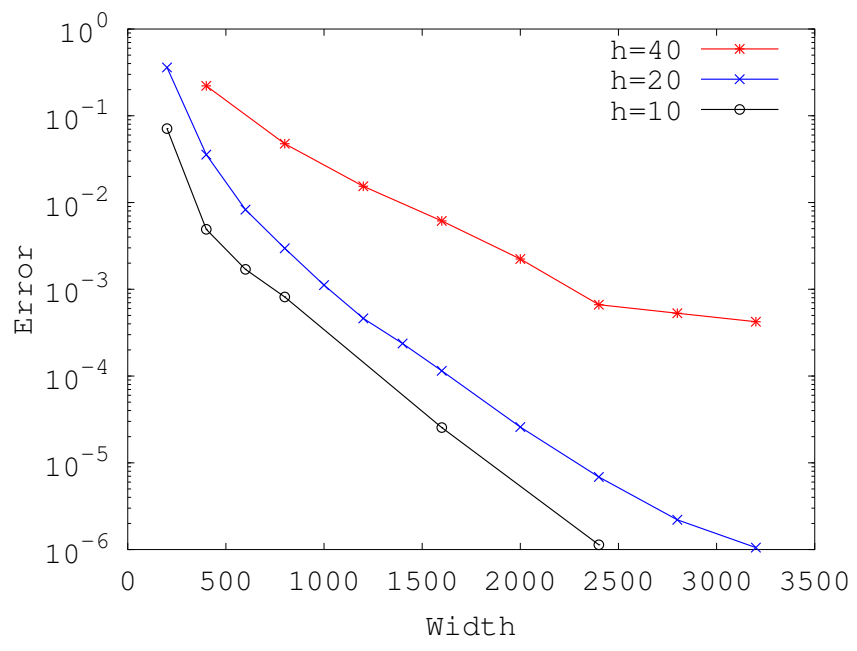

Figure 9: Relative reflection errors as function of the width $\left(h N_{s g}\right)$ of each super-grid layer.

errors of the same order as the propagation errors from the interior scheme. We remark that a similar result was established for Maxwell's equations with PML damping layers [33].

In absolute terms, the required layer width depends on the specific problem. In SW4, the default setting is to use 30 grid points across the layer. This usually gives sufficient accuracy for realistic engineering computations. In the calculations presented in Figure 8, 30 points in the layer results in about $1 \%$ error, while 20 points give $5 \%$ error. These are reasonable tolerances for practical calculations with a fourth order accurate scheme, which often resolve the solution with around 10 grid points per shortest wavelength. We do not recommended using less than 20 grid points across the super-grid layer for two reasons. First because the reflection errors become significant. Secondly, the time stepping scheme might become unstable unless the time step is reduced. This occurs because the super-grid stretching function becomes poorly resolved on the grid and varies significantly from grid point to grid point, leading to additional stiffness of the spatial operator.

\subsection{A half-space problem}

As a final numerical example we study anisotropic wave propagation in a homogeneous InAs crystal subject to a free surface boundary condition 
along $x^{(3)}=0$. We take the domain of interest to be

$$
1.6 \cdot 10^{3} \leq\left(x^{(1)}, x^{(2)}\right) \leq 10.4 \cdot 10^{3}, \quad 0 \leq x^{(3)} \leq 4.4 \cdot 10^{3},
$$

and locate the source term $(69)$ at $\mathbf{x}_{s}=(6,6,1) \cdot 10^{3}$. We pad both sides of the domain of interest by super-grid layers in the $x^{(1)}$ - and $x^{(2)}$-directions. In the $x^{(3)}$-direction, we only add a super-grid layer for $x^{(3)}>4.4 \cdot 10^{3}$. Here, the relative transition width of the damping coefficient profile (66) is $w=1$ and a fourth order artificial dissipation term is used in the super-grid layer. As before, cross-sections of the solution are plotted as function of

scaled parameter coordinates, to equal $\left(x^{(1)}, x^{(2)}, x^{(3)}\right)$ within the domain of interest.

In Figure 10 we show snapshots of the magnitude of the numerical solution with grid size $h=20$, corresponding to 601 grid points in the $x^{(1)}$ and $x^{(2)}$ directions and 301 points in the $x^{(3)}$ direction. There are 80 grid points in each super-grid layer. The solution is shown along the free surface, $x^{(3)}=0$, and in the vertical plane $x^{(1)}=6 \cdot 10^{3}$. Due to the free surface boundary condition along $x^{(3)}=0$, the solution has much more structure compared to the Cauchy problem, and several sets of quasi-compressional, quasi-shear, and surface waves can be identified in the solution. Note that no reflected waves are visible within the domain of interest at time $t=3.5$ (Figure 10, bottom row).

The free surface boundary condition leads to evanescent modes in the solution, i.e., at every fixed location $\mathbf{x}_{r}$, the solution only decays exponentially in time as $t \rightarrow \infty$. Hence, there is no time $t_{1}$ after which the analytical solution is identically zero at $\mathbf{x}_{r}$. Unfortunately, this prevents us from using the same technique as in $\S 6.4$ for quantifying the long time reflection properties of the super-grid layers.

\section{Conclusions}

We have presented a fourth order accurate finite difference discretization of the elastic wave equation in second order formulation for a general, 21 parameter anisotropic, heterogeneous, material. The discretization is defined on a curvilinear grid, by use of a general coordinate transformation. The proposed method generalizes our previous finite difference method [11] to anisotropic elastic materials and curvilinear grids. The proposed method is energy conserving and stable under a CFL time-step constraint, and we have developed a practically useful approach for estimating the size of the largest stable time step. We have also generalized the super-grid technique [12] 

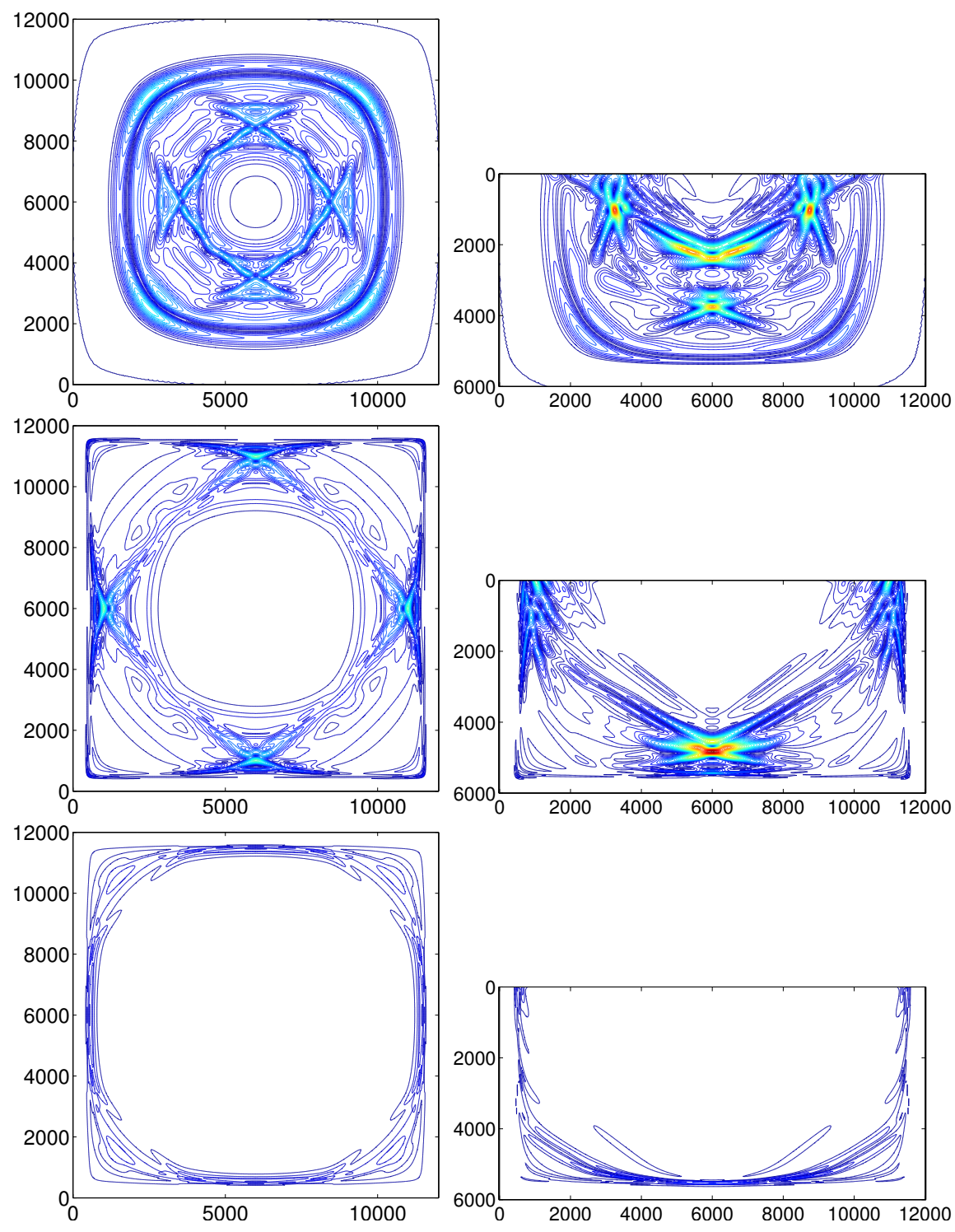

Figure 10: Half-space problem: Magnitude of the displacement at times 1.5, 2.5, and 3.5 (top to bottom) along the free surface $x^{(3)}=0$ (left) and the $x^{(1)}=6 \cdot 10^{3}$ plane (right). In the latter figures, the free surface is located along the top edge. The super-grid layers have thickness $1.6 \cdot 10^{3}$. The contour levels are the same in all plots and are spaced between 0.0375 (dark blue) and 1.5 (red) with step size 0.0375 . 
to anisotropic elastic materials, and demonstrated that it leads to a stable numerical method with very small artificial reflections.

It would be straightforward to extend the proposed method to higher orders of accuracy. Such an extension relies on compatible, higher order accurate, summation by parts operators for approximating both first and second derivatives with variable coefficients. In particular, the difference approximations must satisfy (39) and (41), respectively. For first derivatives, it is well know that such operators exist with up to eighth order truncation error in the interior of the domain, with a reduction to order four on the boundary. For second derivatives with variable coefficients, we have derived operators having truncation errors of order six and eight in the interior, with boundary reduction to order three and four, respectively. Because we solve the elastic wave equation in second order formulation, the solution is two orders more accurate than the truncation error near the boundary. These operators could therefore be used to device a sixth order accurate scheme for the anisotropic elastic wave equation.

By generalizing the technique developed in [34], it would be straightforward to extend the proposed method to include visco-elastic attenuation. However, the number of material parameters would increase by 21 for each visco-elastic mechanism in the model. For the isotropic visco-elastic model, these parameters are usually determined by matching observed attenuation rates of compressional and shear waves [35]. It is unclear if that approach could be generalized to estimate all the parameters in an anisotropic viscoelastic model.

\section{Acknowledgments}

This work performed under the auspices of the U.S. Department of Energy by Lawrence Livermore National Laboratory under contract DE-AC5207NA27344. This is contribution LLNL-JRNL-663238.

\section{Appendix A. The $M^{i j}$ matrices}

The divergence of the stress tensor, $\mathbf{L u}$, can be expressed in terms of the symmetric positive definite $6 \times 6$ stiffness matrix $C$ and the differential 
operator $G_{s}$ in (3),

$$
\mathbf{L u}=G_{s}^{T} C G_{s} \mathbf{u}, \quad C=\left(\begin{array}{cccc}
c_{11} & c_{12} & \cdots & c_{16} \\
c_{12} & c_{22} & \cdots & c_{26} \\
\vdots & \vdots & \ddots & \vdots \\
c_{16} & c_{26} & \cdots & c_{66}
\end{array}\right) .
$$

The divergence of the stress tensor can also be written in the form (7), where the terms are given by (8)-(10). By identifying each term in the two expressions for $\mathbf{L u}$, we arrive at

$$
\begin{aligned}
& M^{11}=\left(\begin{array}{lll}
c_{11} & c_{16} & c_{15} \\
c_{16} & c_{66} & c_{56} \\
c_{15} & c_{56} & c_{55}
\end{array}\right), \quad M^{12}=\left(\begin{array}{lll}
c_{16} & c_{12} & c_{14} \\
c_{66} & c_{26} & c_{46} \\
c_{56} & c_{25} & c_{45}
\end{array}\right), \\
& M^{13}=\left(\begin{array}{lll}
c_{15} & c_{14} & c_{13} \\
c_{56} & c_{46} & c_{36} \\
c_{55} & c_{45} & c_{35}
\end{array}\right), \quad M^{21}=\left(\begin{array}{lll}
c_{16} & c_{66} & c_{56} \\
c_{12} & c_{26} & c_{25} \\
c_{14} & c_{46} & c_{45}
\end{array}\right), \\
& M^{22}=\left(\begin{array}{lll}
c_{66} & c_{26} & c_{46} \\
c_{26} & c_{22} & c_{24} \\
c_{46} & c_{24} & c_{44}
\end{array}\right), \quad M^{23}=\left(\begin{array}{lll}
c_{56} & c_{46} & c_{36} \\
c_{25} & c_{24} & c_{23} \\
c_{45} & c_{44} & c_{34}
\end{array}\right) \text {, } \\
& M^{31}=\left(\begin{array}{lll}
c_{15} & c_{56} & c_{55} \\
c_{14} & c_{46} & c_{45} \\
c_{13} & c_{36} & c_{35}
\end{array}\right), \quad M^{32}=\left(\begin{array}{lll}
c_{56} & c_{25} & c_{45} \\
c_{46} & c_{24} & c_{44} \\
c_{36} & c_{23} & c_{34}
\end{array}\right) \text {, }
\end{aligned}
$$

and

$$
M^{33}=\left(\begin{array}{lll}
c_{55} & c_{45} & c_{35} \\
c_{45} & c_{44} & c_{34} \\
c_{35} & c_{34} & c_{33}
\end{array}\right) .
$$

By inspection, the diagonal blocks $M^{11}, M^{22}$, and $M^{33}$ are symmetric and $M^{j i}=\left(M^{i j}\right)^{T}$ for $i \neq j$. To show that $M^{11}$ is positive definite, we take $\mathbf{z}=\left(z_{1}, z_{2}, z_{3}\right)^{T}$ and $\mathbf{y}=\left(z_{1}, 0,0,0, z_{3}, z_{2}\right)^{T}$. Now,

$$
\mathbf{z}^{T} M^{11} \mathbf{z}=\mathbf{y}^{T} C \mathbf{y} \geq \kappa \mathbf{y}^{T} \mathbf{y}=\kappa \mathbf{z}^{T} \mathbf{z}, \quad \kappa>0,
$$

because $C$ is positive definite. The same technique can be used to show that $M^{22}$ and $M^{33}$ are positive definite. 
[1] G. E. Backus, Long-wave elastic anisotropy produced by horizontal layering, J. Geophys. Res. 67 (1962) 4427-4440.

[2] L. Thompsen, Weak elastic anisotropy, Geophysics 51 (1986) 19541966.

[3] Y. Capdeville, L. Guillot, J.-J. Marigo, 2-D non-periodic homogenization to upscale elastic media for P-SV waves, Geophys. J. Int. 182 (2010) 903-922.

[4] L. Guillot, Y. Capdeville, J.-J. Marigo, 2-D non-periodic homogenization of the elastic wave equation: SH case, Geophys. J. Int. 182 (2010) $1438-1454$.

[5] J. Virieux, P-SV wave propagation in heterogeneous media: Velocitystress finite-difference method, Geophysics 51 (4) (1986) 889-901.

[6] A. Levander, Fourth-order finitie-difference P-SV seismograms, Geophysics 53 (11) (1988) 1425-1436.

[7] R. Graves, Simulating seismic-wave propagation in 3-D elastic media using staggered-grid finite differences, Bull. Seismo. Soc. Amer. 86 (4) (1996) 1091-1106.

[8] D. Komatitsch, J. Tromp, Introduction to the spectral element method for three-dimensional seismic wave propagation, Geophys. J. Int. 139.

[9] M. Chen, J. Tromp, Theoretical and numerical investigations of global and regional seismic wave propagation in weakly anisotropic earth models, Geophys. J. Int. 168 (2007) 1130-1152.

[10] S.-J. Lee, H.-W. Chen, Q. Liu, D. Komatitsch, B.-S. Huang, J. Tromp, Three-dimensional simulations of seismic-wave propagation in the Taipei basin with realistic topography based upon the spectral-element method, Bull. Seismo. Soc. Amer. 98 (1) (2008) 253-264.

[11] B. Sjögreen, N. A. Petersson, A fourth order accurate finite difference scheme for the elastic wave equation in second order formulation, J. Sci. Comput. 52 (2012) 17-48, dOI 10.1007/s10915-011-9531-1.

[12] N. A. Petersson, B. Sjögreen, Super-grid modeling of the elastic wave equation in semi-bounded domains, Comm. Comput. Phys. 16 (2014) 913-955. 
[13] N. A. Petersson, B. Sjögreen, User's guide to SW4, version 1.0, Tech. Rep. LLNL-SM-642292, Lawrence Livermore National Laboratory (2013).

[14] K. Mattsson, Summation by parts operators for finite difference approximations of second-derivative approximations of second-derivatives with variable coefficients, J. Sci. Comput. 51 (2012) 650-682.

[15] K. Duru, G. Kreiss, K. Mattsson, Accurate and stable boundary treatments for elastic wave equations in second order formulation, SIAM J. Sci. Comput. 36 (6) (2014) A2787-A2818.

[16] K. Duru, K. Virta, Stable and high order accurate difference methods for the elastic wave equation in discontinuous media, J. Comput. Phys. 279 (2014) 37-62.

[17] J. P. Berenger, A perfectly matched layer for the absorption of electromagnetic waves, J. Comput. Phys. 114 (1994) 185-200.

[18] E. Bécache, S. Fauqueux, P. Joly, Stability of perfectly matched layers, group velocities and anisotropic waves, J. Comput. Phys. 188 (2003) 399-433.

[19] K. Duru, G. Kreiss, Boundary waves and stability of the perfectly matched layer for the two space dimensional elastic wave equation in second order form, SIAM J. Numer. Anal. 52 (6) (2014) 2883-2904.

[20] J. M. Carcione, Wave fields in real media: wave propagation in anisotropic, anelastic and porous media, Vol. 31 of Handbook of geophysical exploration: seismic exploration, Pergamon, Elsevier Science, 2001.

[21] D. Appelö, N. A. Petersson, A stable finite difference method for the elastic wave equation on complex geometries with free surfaces, Comm. Comput. Phys. 5 (2009) 84-107.

[22] J. F. Thompson, Z. U. Warsi, C. W. Mastin, Numerical grid generation: foundations and applications, Elsevier North-Holland, Inc., New York, NY, USA, 1985.

[23] B. Strand, Summation by parts for finite difference approximations for d/dx, J. Comput. Phys. 110 (1994) 47-67. 
[24] M. Vinokur, H. C. Yee, Extension of efficient low dissipative high order schemes for 3-D curvilinear moving grids, in: D. Caughey, M. Hafez (Eds.), Frontiers of Computational Fluid Dynamics 2002, World Scientific, 2002, pp. 129-164.

[25] B. Sjögreen, H. C. Yee, M. Vinokur, On high order finite-difference metric discretizations satisfying GCL on moving and deforming grids, J. Comput. Phys. 265 (2014) 211-220.

[26] D. A. Kopriva, Metric identities and the discontinuous spectral element method on curvilinear meshes, J. Sci. Comput. 26 (3) (2006) 301-327.

[27] D. Appelö, T. Colonius, A high order super-grid-scale absorbing layer and its application to linear hyperbolic systems, J. Comput. Phys. 228 (2009) 4200-4217.

[28] N. A. Petersson, B. Sjögreen, User's guide to SW4, version 1.1, Tech. Rep. LLNL-SM-662014, Lawrence Livermore National Laboratory, (Source code available from geodynamics.org/cig) (2014).

[29] G. Chesshire, W. D. Henshaw, Composite overlapping meshes for the solution of partial differential equations, J. Comput. Phys. 90 (1) (1990) $1-64$.

[30] W. Martienssen, H. Werner (Eds.), Springer handbook of condensed matter and materials data, Vol. XVIII, Springer, 2005, iSBN 978-3540-44376-6.

[31] N. A. Petersson, B. Sjögreen, Stable grid refinement and singular source discretization for seismic wave simulations, Comm. Comput. Phys. 8 (5) (2010) 1074-1110.

[32] B. Gustafsson, H.-O. Kreiss, J. Oliger, Time Dependent Problems and Difference Methods, John Wiley \& Sons, New York, 1995.

[33] B. Sjögreen, N. A. Petersson, Perfectly matched layers for Maxwell's equations in second order formulation, J. Comput. Phys. 209 (1) (2005) $19-46$.

[34] N. A. Petersson, B. Sjögreen, Stable and efficient modeling of anelastic attenuation in seismic wave propagation, Comm. Comput. Phys. 12 (1) (2012) 193-225. 
[35] H. Emmerich, M. Korn, Incorporation of attenuation into timedependent computations of seismic wave fields, Geophysics 52 (1987) 1252-1264. 